\title{
Consistency of Loop Regularization Method and Divergence Structure of QFTs Beyond One-Loop Order
}

\author{
Da Huang* \\ Kavli Institute for Theoretical Physics China (KITPC) at the CAS \\ State Key Laboratory of Theoretical Physics (SKLTP) \\ Institute of Theoretical Physics, Chinese Academy of Science, Beijing,100190 \\ Department of Physics, National Tsinghua University, Hsinchu, Taiwan 300 \\ Ling-Fong Li才 \\ Kavli Institute for Theoretical Physics China (KITPC) at the CAS \\ Department of Physics, Carnegie Mellon University, Pittsburgh, PA 15213 \\ Yue-Liang $\mathrm{Wu}$ \\ Kavli Institute for Theoretical Physics China (KITPC) at the CAS \\ State Key Laboratory of Theoretical Physics (SKLTP) \\ Institute of Theoretical Physics, Chinese Academy of Science, Beijing,100190 \\ University of Chinese Academy of Sciences
}

(Dated: November 1, 2018)

\begin{abstract}
We study the problem how to deal with tensor-type two-loop integrals in the Loop Regularization (LORE) scheme. We use the two-loop photon vacuum polarization in the massless Quantum Electrodynamics (QED) as the example to present the general procedure. In the processes, we find a new divergence structure: the regulated result for each two-loop diagram contains a gaugeviolating quadratic harmful divergent term even combined with their corresponding counterterm insertion diagrams. Only when we sum up over all the relevant diagrams do these quadratic harmful divergences cancel, recovering the gauge invariance and locality.
\end{abstract}

PACS numbers:

*Electronic address: dahuang@phys.nthu.edu.tw

${ }^{\dagger}$ Electronic address: lfli@cmu.edu

‡Electronic address: ylwu@itp.ac.cn 


\section{INTRODUCTION}

This is the second of a series of papers to study the consistency of Loop Regularization (LORE) scheme and the general structure of QFT models in the higher-loop calculations. In our first paper[1], we have shown that the LORE method is consistent for general scalar-type two-loop integrals. We studied the general $\alpha \beta \gamma$ integrals[2] and mainly solved the problem of disentangling the overlapping divergences, which is the main new feature of the perturbative calculations beyond one-loop order. We argued that the overlapping divergence structure would, in general, transforms some of the ultraviolet (UV) divergences into the integrals of the ultraviolet divergence-preserving(UVDP) parameters (or Feynman parameters). We found that the LORE method was particularly suitable to regularize the UV divergences of this kind since we can easily transform the UVDP parameter integrals into the irreducibleloop-integral(ILI)-like integrals by multiplying the integration variable by a mass scale, which is exactly the object regulated in the LORE method. Furthermore, it was very useful to introduce the Bjorken and Drell's analogy between the Feynman diagrams and electric circuits, which allowed us to identify the UV divergences of the UVDP parameters with those contained in the subdiagrams of the original Feynman diagrams. Then we applied all the tools we found to the study of two-loop calculations of the $\phi^{4}$ model and obtained the consistent results for $\beta$-function of the coupling constant and mass parameter. Finally, we gave the general procedure of the application of the LORE method to the higher-loop calculations, even beyond two-loop order.

In the present paper, we will focus on one of the important remaining problems- how to deal with the tensor-type integrals at higher-loop order. Recall that at one-loop order in LORE tensor-type ILIs and scalar-type ILIs can be related by introducing consistency conditions, which relate these type of integrals with the same divergent behavior. So the immediate question is whether these consistent conditions, which should be applied to the 1-folded ILIs of each loop momentum variable, is still consistent with the general features we find in our previous paper. In particular, we want to ask whether the consistent conditions can guarantee the cancellation of various harmful divergences. Another question involves the gauge invariance which is ensured by the consistency conditions in LORE as shown in $[3,4]$. Thus, it is necessary to inquire whether the consistency conditions can still preserve gauge invariance and the associated Ward identities. In order to study these questions, we use the massless Quantum Electrodynamics (QED) as our simplest example, which involves the tensor-type integrals and the gauge invariance at the same time. Specifically, we shall calculate the two-loop photon vacuum polarization diagrams in detail. As will be shown in the following calculation, the LORE method, with the prescription of consistency conditions, can give the sensible results which satisfy all the requirements: gauge invariance and locality. We should emphasize that the result above only requires the use of consistency conditions found at one-loop order and does not need to introduce additional two-loop order consistency conditions.

One special feature worth mentioning is the appearance of a new divergence structure: each two-loop Feynman diagram contains a quadratically harmful divergence even when we 
combine the result with the corresponding one-loop counterterm insertion diagrams. These new divergence structure is expected and compatible with the usual power counting for each diagrams. However, these quadratically harmful divergences will cancel with each other when we sum up all three group of diagrams in this order, rending the final result local. Another way to see this cancellation can be obtained from the requirement of gauge invariance of the final result since these quadratically harmful divergences break the gauge invariance and must disappear in the final result. We argue that this is a general feature for gauge theories, since quadratic divergences would in general give the photon a mass, which is incompatible with gauge invariance. The appearance of this new divergence structure explicitly shows one of the main advantage of LORE method that the LORE method enables us to calculate the full result for any Feynman diagram, especially the quadratic divergences, which is absent in many other regularization methods, like the popular dimensional regularization (DR). In the light of detailed and complete calculations, we conclude that the LORE method can properly regularize tensor-type high-loop integrals (at least up to two-loop) and consistently apply to general gauge theories, like the Standard Model (SM) of particle physics.

The paper is organized as follows. Section 2 gives a short introduction to the LORE method. In Section 3, we first compute all of the one-loop one-particle-irreducible diagrams that need to be regulated, in order to set our notations. Then we present the results of the massless QED vacuum polarization at two-loop order by using the LORE method, leaving the many of the details to the Appendix B and C. Appendix A contains some useful formulae in the LORE method. Appendix D contributes to the detailed calculation of a new doublylogarithmic divergent integral encountered in our two-loop calculations.

\section{IRREDUCIBLE LOOP INTEGRALS(ILIS) AND THE PRESCRIPTION OF LOOP REGULARIZATION (LORE)}

In this section, we give a brief introduction to the Loop Regularization (LORE). A more detailed discussion will be found in the seminal paper by one of the authors [3].

It has been shown in [3, 4] that all one loop Feynman integrals can be reduced into the following 1-fold ILIs by using the standard Feynman parameterization method:

$$
\begin{aligned}
I_{-2 \alpha} & =\int \frac{d^{4} k}{(2 \pi)^{4}} \frac{1}{\left(k^{2}-M^{2}\right)^{2+\alpha}}, \\
I_{-2 \alpha \mu \nu} & =\int \frac{d^{4} k}{(2 \pi)^{4}} \frac{k_{\mu} k_{\nu}}{\left(k^{2}-M^{2}\right)^{3+\alpha}}, \quad \alpha=-1,0,1,2, \ldots \\
I_{-2 \alpha \mu \nu \rho \sigma} & =\int \frac{d^{4} k}{(2 \pi)^{4}} \frac{k_{\mu} k_{\nu} k_{\rho} k_{\sigma}}{\left(k^{2}-M^{2}\right)^{4+\alpha}}
\end{aligned}
$$

with $I_{2}$ and $I_{0}$ corresponding to the quadratic and logarithmic divergent integrals. Here the effective mass factor $M^{2}$ is a function of the external momenta $p_{i}$, the masses of particles $m_{i}$ and the Feynman parameters.

When the regularized 1-fold ILIs satisfy the following consistency conditions [3, 4] :

$$
\begin{array}{ll}
I_{2 \mu \nu}^{R}=\frac{1}{2} g_{\mu \nu} I_{2}^{R}, & I_{2 \mu \nu \rho \sigma}^{R}=\frac{1}{8}\left(g_{\mu \nu} g_{\rho \sigma}+g_{\mu \rho} g_{\nu \sigma}+g_{\mu \sigma} g_{\rho \nu}\right) I_{2}^{R}, \\
I_{0 \mu \nu}^{R}=\frac{1}{4} g_{\mu \nu} I_{0}^{R}, & I_{0 \mu \nu \rho \sigma}^{R}=\frac{1}{24}\left(g_{\mu \nu} g_{\rho \sigma}+g_{\mu \rho} g_{\nu \sigma}+g_{\mu \sigma} g_{\rho \nu}\right) I_{0}^{R} .
\end{array}
$$

the resulting loop corrections are gauge invariant. Here the superscript "R" denotes the regularized ILIs. 
Note that the introduction of the irreducible loop integrals (ILIs) is crucial in the loop regularization [3, 4], and it has been shown that all Feynman loop integrals can be expressed in terms of the ILIs. In the definition of ILIs, one important feature is that there should be no factor of $k^{2}$ in the numerator of loop integration and all the ILIs can be classified into the scalar type ILIs with the following loop integrand

$$
\frac{1}{\left(k^{2}-M^{2}\right)^{\alpha}}
$$

and the tensor type ILIs with the following loop integration

$$
\frac{k_{\mu} k_{\nu} \cdots k_{\rho}}{\left(k^{2}-M^{2}\right)^{\alpha}}
$$

In manipulating the Feynman loop integrals into ILIs, one should always perform the Dirac algebra and Lorentz index-contraction first to obtain the ILIs defined by the above "simplest" forms for the one loop case. For example, the integrand

$$
g^{\mu \nu} \cdot k_{\mu} k_{\nu} /\left(k^{2}-M^{2}\right)^{2}
$$

should not be written as

$$
g^{\mu \nu} \cdot I_{2 \mu \nu}
$$

Instead it should be expressed as

$$
k^{2} /\left(k^{2}-M^{2}\right)^{2}
$$

and then rewrite the $k^{2}$ in the numerator in the form $\left(k^{2}-M^{2}\right)+M^{2}$ so as to cancel out the first term by the denominator to get,

$$
g^{\mu \nu} \cdot k_{\mu} k_{\nu} /\left(k^{2}-M^{2}\right)^{2}=I_{2}+M^{2} \cdot I_{0} .
$$

A simple regularization prescription for the ILIs was realized to yield the above consistency conditions. The procedure is: rotating to the four dimensional Euclidean space of momentum, replacing the loop integrating variable $k^{2}$ and the loop integrating measure $\int d^{4} k$ in the ILIs by the corresponding regularized ones $\left[k^{2}\right]_{l}$ and $\int\left[d^{4} k\right]_{l}$ :

$$
\begin{aligned}
k^{2} & \rightarrow\left[k^{2}\right]_{l} \equiv k^{2}+M_{l}^{2} \\
\int d^{4} k & \rightarrow \int\left[d^{4} k\right]_{l} \equiv \lim _{N, M_{l}^{2}} \sum_{l=0}^{N} c_{l}^{N} \int d^{4} k
\end{aligned}
$$

where $M_{l}^{2}(l=0,1, \cdots)$ may be regarded as the regulator masses for the ILIs. The regularized ILIs in the Euclidean space-time are then given by:

$$
\begin{aligned}
I_{-2 \alpha}^{R} & =i(-1)^{\alpha} \lim _{N, M_{l}^{2}} \sum_{l=0}^{N} c_{l}^{N} \int \frac{d^{4} k}{(2 \pi)^{4}} \frac{1}{\left(k^{2}+M^{2}+M_{l}^{2}\right)^{2+\alpha}}, \\
I_{-2 \alpha \mu \nu}^{R} & =-i(-1)^{\alpha} \lim _{N, M_{l}^{2}} \sum_{l=0}^{N} c_{l}^{N} \int \frac{d^{4} k}{(2 \pi)^{4}} \frac{k_{\mu} k_{\nu}}{\left(k^{2}+M^{2}+M_{l}^{2}\right)^{3+\alpha}}, \quad \alpha=-1,0,1,2, \ldots \\
I_{-2 \alpha \mu \nu \rho \sigma}^{R} & =i(-1)^{\alpha} \lim _{N, M_{l}^{2}} \sum_{l=0}^{N} c_{l}^{N} \int \frac{d^{4} k}{(2 \pi)^{4}} \frac{k_{\mu} k_{\nu} k_{\rho} k_{\sigma}}{\left(k^{2}+M^{2}+M_{l}^{2}\right)^{4+\alpha}}
\end{aligned}
$$


where the coefficients $c_{l}^{N}$ are chosen to satisfy the following conditions:

$$
\lim _{N, M_{l}^{2}} \sum_{l=0}^{N} c_{l}^{N}\left(M_{l}^{2}\right)^{n}=0 \quad(n=0,1, \cdots)
$$

with the notation $\lim _{N, M_{l}^{2}}$ denoting the $\operatorname{limit} \lim _{N, M_{R}^{2} \rightarrow \infty}$. One may take the initial conditions $M_{0}^{2}=\mu_{s}^{2}=0$ and $c_{0}^{N}=1$ to recover the original integrals in the limit $M_{l}^{2} \rightarrow \infty(l=1,2, \cdots$ ). Such a new regularization is called as Loop Regularization (LORE) [3, 4]. The prescription in LORE method is very similar to Pauli-Villars prescription, but two concepts are totally different as the prescription in the loop regularization is acting on the ILIs rather than on the propagators as in Pauli-Villars scheme. This is why the Pauli-Villars regularization violates non-Abelian gauge symmetry, while LORE method can preserve non-Abelian gauge symmetry.

A simple solution of eq. (5), is to take the string-mode regulators,

$$
M_{l}^{2}=\mu_{s}^{2}+l M_{R}^{2}
$$

with $l=1,2, \cdots$, and the coefficients $c_{l}^{N}$ to be of the form,

$$
c_{l}^{N}=(-1)^{l} \frac{N !}{(N-l) ! l !}
$$

Here $M_{R}$ may be regarded as a basic mass scale of loop regulator . It has been shown in [4] that the above regularization prescription can be understood in terms of Schwinger proper time formulation with an appropriate regulating distribution function.

With the string-mode regulators for $M_{l}^{2}$ and $c_{l}^{N}$ in above equations, the regularized ILIs $I_{2}^{R}$ and $I_{0}^{R}$ can be calculated to give[3, 4]:

$$
\begin{aligned}
I_{2}^{R} & =\frac{-i}{16 \pi^{2}}\left\{M_{c}^{2}-\mu^{2}\left[\ln \frac{M_{c}^{2}}{\mu^{2}}-\gamma_{w}+1+y_{2}\left(\frac{\mu^{2}}{M_{c}^{2}}\right)\right]\right\} \\
I_{0}^{R} & =\frac{i}{16 \pi^{2}}\left[\ln \frac{M_{c}^{2}}{\mu^{2}}-\gamma_{w}+y_{0}\left(\frac{\mu^{2}}{M_{c}^{2}}\right)\right]
\end{aligned}
$$

with $\mu^{2}=\mu_{s}^{2}+M^{2}$, and

$$
\begin{aligned}
& \gamma_{w} \equiv \lim _{N}\left\{\sum_{l=1}^{N} c_{l}^{N} \ln l+\ln \left[\sum_{l=1}^{N} c_{l}^{N} l \ln l\right]\right\}=\gamma_{E}=0.5772 \cdots, \\
& y_{0}(x)=\int_{0}^{x} d \sigma \frac{1-e^{-\sigma}}{\sigma}, \quad y_{1}(x)=\frac{e^{-x}-1+x}{x} \\
& y_{2}(x)=y_{0}(x)-y_{1}(x), \quad \lim _{x \rightarrow 0} y_{i}(x) \rightarrow 0, i=0,1,2 \\
& M_{c}^{2} \equiv \lim _{N, M_{R}} M_{R}^{2} \sum_{l=1}^{N} c_{l}^{N}(l \ln l)=\lim _{N, M_{R}} M_{R}^{2} / \ln N
\end{aligned}
$$

This indicates that the $\mu_{s}$ sets an IR 'cutoff' at $M^{2}=0$ and $M_{c}$ provides an UV 'cutoff'. For renormalizable quantum field theories, $M_{c}$ can be taken to infinity $\left(M_{c} \rightarrow \infty\right)$. In a theory without infrared divergence, $\mu_{s}$ can safely run to $\mu_{s}=0$. Actually, in the case that $M_{c} \rightarrow \infty$ and $\mu_{s}=0$, one recovers the initial integral. Also once $M_{R}$ and $N$ are taken to be infinity, the regularized theory becomes independent of the regularization prescription. Note that to evaluate the ILIs, products of $\gamma$ matrices involving loop momentum $\not k$ such as $\not k \gamma_{\mu} \not k$ should be reduced to one of the independent components: $\gamma_{\mu}, \sigma_{\mu \nu}, \gamma_{5} \gamma_{\mu}, \gamma_{5}$ without $k$. 


\section{MASSLESS QED VACUUM POLARIZATION AT TWO LOOP ORDER}

In this section, we will present our results of vacuum polarization in the massless Quantum Electrodynamics (QED) at two-loop order. The motivation for this computation is twofolded: (1) to show how to apply Loop Regularization (LORE) method to tensor-type twoloop integrals; (2) to give the first explicit example of applying LORE to the realistic model. We will show that LORE can preserve Ward Identity and in turn the gauge invariance of QED at two-loop order. Also, our result reproduces the results in the standard textbooks like [5-7]. It is an essential step towards applying LORE to the general gauge theory calculations, such as the Standard Model of particle physics.

The Lagrangian of the massless QED is:

$$
\mathcal{L}_{Q E D}=-\frac{1}{4}\left(F_{\mu \nu}\right)^{2}+i \bar{\psi} D \psi
$$

where, $D_{\mu}=\partial_{\mu}+i e A_{\mu}$ is the covariant derivative and $F_{\mu \nu}=\partial_{\mu} A_{\nu}-\partial_{\nu} A_{\mu}$ is the field strength tensor of electromagnetic field. The detailed Feynman rules for QED are referred to the standard texts, such as [ $[-7]$.

\section{A. Regularization and Renormalization of QED at One-Loop Level}

In this section, we present the regularization and renormalization for all the divergent oneparticle-irreducible (1PI) diagrams in the massless QED at one-loop level with the LORE for use in the later calculation. At one-loop level, the massless QED has three divergent 1PI diagrams requiring regularization and renormalization. They are shown in Fig.(11)

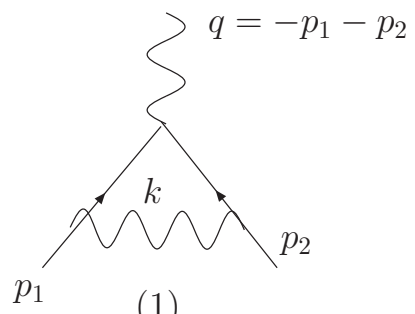

(1)

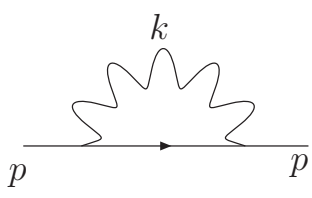

(2)

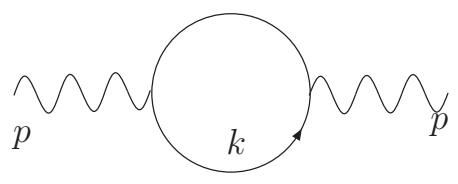

(3)

FIG. 1: 1-Loop 1PI diagrams: Left: Vertex correction; Middle: Electron self energy; Right: Vacuum Polarization

Using the standard method of Feynman parameters, we get for the vertex correction

$$
\begin{aligned}
-i \Gamma^{\mu(1)} & =(-i e)^{3} \int \frac{d^{4} k}{(2 \pi)^{4}}\left(\gamma^{\sigma} \frac{i}{\not p+\not p_{2}} \gamma^{\mu} \frac{i}{\not k-\not p_{1}} \gamma^{\rho}\right) \frac{-i g_{\rho \sigma}}{k^{2}} \\
& =2 \Gamma(3) e^{3} \int d x d y \int \frac{d^{4} k}{(2 \pi)^{4}} \frac{\left[\not p-(1-x) \not p_{1}-y \not \not_{2}\right] \gamma^{\mu}\left[\not k+x \not \not_{1}+(1-y) \not p_{2}\right]}{\left[k^{2}-M_{p_{1}, p_{2}}^{2}\right]^{3}}
\end{aligned}
$$

where $M_{p_{1}, p_{2}}^{2}=\left(x p_{1}-y p_{2}\right)^{2}-x p_{1}^{2}-y p_{2}^{2}$. We can write this in terms of 1 -folded ILIs as,

$$
\begin{aligned}
-i \Gamma^{\mu(1)} & =2 \Gamma(3) e^{3} \int d x d y\left\{\left(\gamma^{\alpha} \gamma^{\mu} \gamma^{\beta}\right) I_{0 \alpha \beta}^{R}-\left[(1-x) \not p_{1}+y \not p_{2}\right] \gamma^{\mu}\left[x \not p_{1}+(1-y) \not p_{2}\right] I_{-2}\right\} \\
& =2 \Gamma(3) e^{3} \int d x d y\left\{-\frac{1}{2} \gamma^{\mu} I_{0}^{R}-\left[(1-x) \not \not_{1}+y \not p_{2}\right] \gamma^{\mu}\left[x \not p_{1}+(1-y) \not p_{2}\right] I_{-2},\right\}
\end{aligned}
$$


where in the second line we have applied the consistency condition Eq.(2) to deal with the regularized logarithmic divergent integral $I_{0 \mu \nu}^{R}$. By using the explicit expression of $I_{0}^{R}$ Eq. (8) and integrating out $I_{-2}$, we can write our result of the one-loop vertex correction as,

$$
-i \Gamma^{\mu(1)}=-\frac{i e^{3}}{8 \pi^{2}} \int d x d y\left\{\gamma^{\mu}\left[\ln \frac{M_{c}^{2}}{\mu_{1}^{2}}-\gamma_{\omega}+y_{0}\left(\frac{\mu_{1}^{2}}{M_{c}^{2}}\right)\right]-\frac{\left[(1-x) \not \phi_{1}+y \not \phi_{2}\right] \gamma^{\mu}\left[x \not \phi_{1}+(1-y) \not p_{2}\right]}{\left(x p_{1}-y p_{2}\right)^{2}-x p_{1}^{2}-y p_{2}^{2}}\right\},
$$

where $\mu_{1}^{2}=\mu_{s}^{2}+M_{p_{1}, p_{2}}^{2}$. According to our renormalization scheme in LORE method discussed in our first paper[1], we should define the one-loop vertex counterterm as:

$$
-i e \gamma^{\mu} \delta_{1}=-\frac{i e^{3}}{8 \pi^{2}} \gamma^{\mu} \int d x d y\left(\ln \frac{M_{c}^{2}}{\mu^{2}}-\gamma_{\omega}\right)=-\frac{i e^{3}}{16 \pi^{2}} \gamma^{\mu}\left(\ln \frac{M_{c}^{2}}{\mu^{2}}-\gamma_{\omega}\right)
$$

where $\mu$ is introduced as the renormalization scale. With such definition of $\delta_{1}$, the renormalized vertex correction at one-loop level is:

$$
-i \Gamma_{R}^{\mu(1)}=-\frac{i e^{3}}{8 \pi^{2}} \int d x d y\left\{\gamma^{\mu} \ln \frac{\mu^{2}}{\mu_{1}^{2}}-\frac{\left[(1-x) \not \phi_{1}+y \not p_{2}\right] \gamma^{\mu}\left[x \not \phi_{1}+(1-y) \not \phi_{2}\right]}{\left(x p_{1}-y p_{2}\right)^{2}-x p_{1}^{2}-y p_{2}^{2}}\right\},
$$

where we have taken the limit $M_{c}^{2} \rightarrow \infty$ so that $y_{0}\left(\frac{\mu_{1}^{2}}{M_{c}^{2}}\right) \rightarrow 0$ to simplify our final result.

For the electron self-energy diagram, we can use the same procedure while the calculation is more straightforward,

$$
\begin{aligned}
i \not(p) & =(-i e)^{2} \int \frac{d^{4} k}{(2 \pi)^{4}}\left(\gamma^{\mu} \frac{i}{\not p+\not p} \gamma^{\nu}\right) \frac{-i g_{\mu \nu}}{k^{2}} \\
& =2 e^{2} \int_{0}^{1} d x \int \frac{d^{4} k}{(2 \pi)^{4}} \frac{(1-x) \not p}{\left[k^{2}+x(1-x) p^{2}\right]^{2}}
\end{aligned}
$$

Using the general formula of LORE, we can regularize the above logarithmic divergent integral as follows:

$$
\begin{aligned}
i \Sigma(p) & =2 e^{2} \not p \int_{0}^{1} d x(1-x) I_{0}^{R} \\
& =\frac{i e^{2}}{8 \pi^{2}} \not p \int_{0}^{1} d x(1-x)\left[\ln \frac{M_{c}^{2}}{\mu_{s}^{2}-x(1-x) p^{2}}-\gamma_{\omega}+y_{0}\left(\frac{\mu_{s}^{2}-x(1-x) p^{2}}{M_{c}^{2}}\right)\right] .
\end{aligned}
$$

If we introduce the counterterm for the self-energy as:

$$
i \not p \delta_{2}=-\frac{i e^{2}}{16 \pi^{2}} \not p\left(\ln \frac{M_{c}^{2}}{\mu}-\gamma_{\omega}\right),
$$

then the renormalized self-energy for electron is:

$$
i \Psi_{R}(p)=\frac{i e^{2}}{8 \pi^{2}} \not p \int_{0}^{1} d x(1-x) \ln \frac{\mu^{2}}{\mu_{s}^{2}-x(1-x) p^{2}},
$$

Note that the counterterm coefficients defined above have the interesting relationship $\delta_{1}=\delta_{2}$, which is the result of Ward identity in QED. This relationship is crucial to guarantee the consistency of our later discussion of renormalization at two-loop level. 
Finally, the one-loop vacuum polarization diagram can be written as:

$$
\begin{aligned}
\mathcal{M}^{(1)} & =(-1)(-i e)^{2} \int \frac{d^{4} k}{(2 \pi)^{4}} \operatorname{tr}\left(\gamma^{\mu} \frac{i}{\not k} \gamma^{\nu} \frac{i}{\not k+\not p}\right) \\
& =-e^{2} \operatorname{tr}\left(\gamma^{\mu} \gamma^{\alpha} \gamma^{\nu} \gamma^{\beta}\right) \int \frac{d^{4} k}{(2 \pi)^{4}} \int_{0}^{1} d x \frac{k_{\alpha} k_{\beta}-x(1-x) p_{\alpha} p_{\beta}}{\left[k^{2}+x(1-x) p^{2}\right]^{2}}
\end{aligned}
$$

By computing the trace of gamma matrices, we obtain the following expression in terms of ILIs:

$$
\mathcal{M}^{(1)}=-4 e^{2} \int_{0}^{1} d x\left\{\left(2 I_{2}^{\mu \nu R}-g^{\mu \nu} I_{2}^{R}\right)-2 x(1-x)\left(p^{\mu} p^{\nu}-g^{\mu \nu} p^{2}\right) I_{0}^{R}\right\} .
$$

With help of consistency condition Eq.(2), it is seen that the quadratically divergent terms in the first parenthesis cancel each other exactly, and the remaining term is proportional to $\left(p^{\mu} p^{\nu}-g^{\mu \nu} p^{2}\right)$, as required by gauge invariance. Using the formula for $I_{0}^{R}$, we get,

$$
\mathcal{M}^{(1)}=\frac{i e^{2}}{2 \pi^{2}}\left(p^{\mu} p^{\nu}-g^{\mu \nu} p^{2}\right) \int_{0}^{1} d x x(1-x)\left[\ln \frac{M_{c}^{2}}{\mu_{s}^{2}-x(1-x) p^{2}}-\gamma_{\omega}+y_{0}\left(\frac{\mu_{s}-x(1-x) p^{2}}{M_{c}^{2}}\right)\right] .
$$

Again, if we choose the counterterm for the photon vacuum polarization as:

$$
\begin{aligned}
i\left(p^{\mu} p^{\nu}-g^{\mu \nu} p^{2}\right) \delta_{3} & =\frac{i e^{2}}{2 \pi^{2}}\left(p^{\mu} p^{\nu}-g^{\mu \nu} p^{2}\right) \int_{0}^{1} d x x(1-x)\left(\ln \frac{M_{c}^{2}}{\mu^{2}}-\gamma_{\omega}\right) \\
& =\frac{i e^{2}}{12 \pi^{2}}\left(p^{\mu} p^{\nu}-g^{\mu \nu} p^{2}\right)\left(\ln \frac{M_{c}^{2}}{\mu^{2}}-\gamma_{\omega}\right)
\end{aligned}
$$

the renormalized photon vacuum polarization at one-loop level is:

$$
\mathcal{M}^{(1)}=\frac{i e^{2}}{2 \pi^{2}}\left(p^{\mu} p^{\nu}-g^{\mu \nu} p^{2}\right) \int_{0}^{1} d x x(1-x) \ln \frac{\mu^{2}}{\mu_{s}^{2}-x(1-x) p^{2}},
$$

where we have taken $y_{0}\left(\frac{\mu_{s}^{2}-x(1-x) p^{2}}{M_{c}^{2}}\right) \rightarrow 0$ when $M_{c}^{2} \rightarrow \infty$ as usual. This completes our discussion of the regularization and renormalization of massless QED at one-loop level.

\section{B. Self-Energy Insertion Diagrams}

Now we compute the photon vacuum polarization at two-loop order. In this subsection, we calculate the diagrams $\left(a_{1}\right)$ and $\left(a_{2}\right)$. It is easy to see that the two diagrams are equal and can be recognized as the insertion of one-loop electron self-energy into the one-loop photon vacuum polarization.

We first write 1-loop electron self-energy using the UVDP parametrization, rather than the Feynman parametrization like we did in Sec.IIIA. The reason lies in the fact that this time the one-loop electron self-energy appears as the subdiagram in $\left(a_{1}\right)$ and may contribute to the overlapping divergence in the following calculation,

$$
\begin{aligned}
i \not^{(1)}\left(k_{1}\right) & =(-i e)^{2} \int \frac{d^{4} k_{2}}{(2 \pi)^{4}}\left(\gamma^{\mu} \frac{i}{\not k_{1}+\not k_{2}} \gamma^{\nu}\right) \frac{-i g_{\mu \nu}}{k_{2}^{2}} \\
& =2 e^{2} \int_{0}^{\infty} \frac{d u u}{(u+1)^{3}} \int \frac{d^{4} k_{2}}{(2 \pi)^{4}} \frac{\not k_{1}}{\left[k_{2}^{2}+\frac{u}{(1+u)^{2}} k_{1}^{2}\right]^{2}},
\end{aligned}
$$



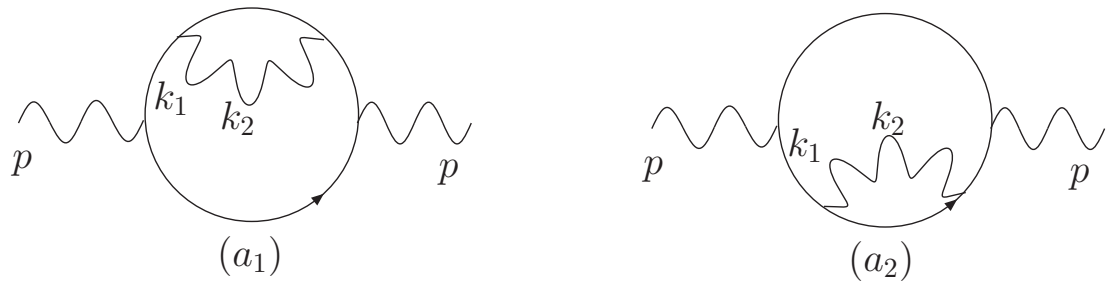

FIG. 2: Self-Energy Insertion Diagrams. Left: $\left(a_{1}\right)$; Right: $\left(a_{2}\right)$

By inserting the above result into the expression of one-loop photon vacuum polarization diagram, we obtain:

$$
\begin{aligned}
\mathcal{M}^{\left(a_{1}\right)}= & (-i e)^{2} \int \frac{d^{4} k_{1}}{(2 \pi)^{4}}(-1) \operatorname{tr}\left[\gamma^{\mu} \frac{i}{\not \phi_{1}} i \Psi^{(1)}\left(k_{1}\right) \frac{i}{\not \phi_{1}} \gamma^{\nu} \frac{i}{\not k_{1}+\not p^{\prime}}\right] \\
= & -2 i e^{4} \int_{0}^{\infty} \frac{d u(u+1)}{u} \int \frac{d^{4} k_{1}}{(2 \pi)^{4}} \int \frac{d^{4} k_{2}}{(2 \pi)^{4}} \operatorname{tr}\left(\gamma^{\mu} \gamma^{\rho} \gamma^{\nu} \gamma^{\sigma}\right) \int_{0}^{1} d x \\
& \frac{k_{1 \rho}\left(k_{1}+p\right)_{\sigma}}{\left[\frac{(1+u)^{2}}{u} k_{2}^{2}+k_{1}^{2}\right]^{2}\left[k_{1}^{2}+2 x k_{1} p+x p^{2}\right]^{2}} .
\end{aligned}
$$

Note that we have used the usual Feynman parameter $x$ to combine the denominators involving $k_{1}$ only, rather than combine all of the three factors once at all. This transforms the Feynman integral $\mathcal{M}^{\left(a_{1}\right)}$ into the $\alpha \beta \gamma$-like integrals (we have already combine two factors in Eq.(18) with UVDP parameter $u$ ). The advantage of this procedure is that it effectively separates the UV divergences from the IR ones in the parameter space. To see this more explicitly, our first paper[1] already explicitly showed that the $\alpha \beta \gamma$ integrals contain the most general UV divergence structure. In other words, the UV divergence can only be contained in $\alpha \beta \gamma$ integrals and their corresponding UVDP parameter integrals, rather in the Feynman parameter space. If we find some divergence in integrating Feynman parameter $x$, then it must be the IR divergence, rather than the UV one. Since we have already written the integral into a generalized $\alpha \beta \gamma$ form, for the rest factors, we will apply the UVDP parameters to combine them,

$$
\begin{aligned}
\mathcal{M}^{\left(a_{1}\right)}= & -8 i e^{4} \Gamma(4) \int_{0}^{\infty} \frac{d u(1+u)}{u} \int_{0}^{1} d x \int_{0}^{\infty} \frac{d v_{1} v_{1}}{\left(1+v_{1}\right)^{4}} \int \frac{d^{4} k_{1}}{(2 \pi)^{4}} \int \frac{d^{4} k_{2}}{(2 \pi)^{4}} \\
& \frac{\left(2 k_{1}^{\mu} k_{1}^{\nu}-g^{\mu \nu} k_{1}^{2}\right)-\frac{x v_{1}}{1+v_{1}}\left(1-\frac{x v_{1}}{1+v_{1}}\right)\left(2 p^{\mu} p^{\nu}-g^{\mu \nu} p^{2}\right)}{\left[k_{1}^{2}+\frac{x v_{1}}{1+v_{1}}\left(1-\frac{x v_{1}}{1+v_{1}}\right) p^{2}+\frac{(1+u)^{2}}{u\left(1+v_{1}\right)} k_{2}^{2}\right]^{4}}
\end{aligned}
$$

Note also that, in the above calculation, we used the traditional technique of completing squares for each internal loop momentum and then shifting the origin of it in order to eliminate the terms linear in the momenta. The resulting formula appears less symmetric in the UVDP variables than that with the general formulae in Ref.[1]. However, this traditional method is more flexible and more natural in practice when the numerator is a complicated function of internal and external momenta like the present case, rather than a constant for the scalar integrals. In the following calculation of Fig. (41), we will also use this traditional method. 
Since there is no cross terms between $k_{1}$ and $k_{2}$ in the denominator, we can easily integrate out $k_{1}$ and $k_{2}$ with the LORE method sequentially:

$$
\begin{aligned}
\mathcal{M}^{\left(a_{1}\right)}= & \frac{8 i e^{4} \Gamma(4)}{16 \pi^{2}} \int_{0}^{\infty} \frac{d u(1+u)}{u} \int_{0}^{1} d x \int_{0}^{\infty} \frac{d v_{1} v_{1}}{\left(1+v_{1}\right)^{4}}\left\{\frac{1}{6} g^{\mu \nu} \frac{u\left(1+v_{1}\right)}{(1+u)^{2}} I_{2}^{R}\left(\mu^{2}\right)\right. \\
& \left.-\frac{1}{6}\left(2 p^{\mu} p^{\nu}-g^{\mu \nu} p^{2}\right) \frac{x v_{1}}{1+v_{1}}\left(1-\frac{x v_{1}}{1+v_{1}}\right) \frac{u^{2}\left(1+v_{1}\right)^{2}}{(1+u)^{4}} I_{0}^{R}\left(\mu^{2}\right)\right\} \\
= & \mathcal{M}_{2}^{\left(a_{1}\right)}+\mathcal{M}_{0}^{\left(a_{1}\right)},
\end{aligned}
$$

where $\mu^{2} \equiv \mu_{s}^{2}-\frac{u x v_{1}}{(1+u)^{2}}\left(1-\frac{x v_{1}}{1+v_{1}}\right) p^{2}$ and $\mathcal{M}_{2(0)}^{\left(a_{1}\right)}$ denotes quadratic (logarithmic) divergent part from the integration of $k_{2}$.

Note that since our integrals in the present paper do not contain any IR divergences, we can set $\mu_{s}^{2} \rightarrow 0$ which also plays the role of IR regulator in the LORE method. Moreover, because the $y_{i}\left(\frac{\mu^{2}}{M_{c}^{2}}\right) \rightarrow 0$ in the limit of $\frac{\mu^{2}}{M_{c}^{2}} \rightarrow 0$, so these functions do not contribute to our final results and can be ignored.

To make our discussion more concise, we only present our final regulated result for the diagram $\left(a_{1}\right)$, while relegating our calculational details of the UVDP parameter integrations to the Appendix B.

$$
\begin{aligned}
\mathcal{M}^{\left(a_{1}\right)} \sim & \frac{4 i e^{4}}{\left(16 \pi^{2}\right)^{2}}\left\{g^{\mu \nu} M_{c}^{2}\left(\ln \frac{M_{c}^{2}}{-q_{o}^{2}}-\gamma_{\omega}\right)+\frac{1}{6} g^{\mu \nu} p^{2}\left(\ln \frac{M_{c}^{2}}{-q_{o}^{2}}-\gamma_{\omega}\right)\right. \\
& +\left(g^{\mu \nu} p^{2}-p^{\mu} p^{\nu}\right) \cdot\left[\frac{1}{3}\left(\ln \frac{M_{c}^{2}}{-p^{2}}-\gamma_{\omega}\right)\left(\ln \frac{M_{c}^{2}}{-q_{o}^{2}}-\gamma_{\omega}\right)-\frac{1}{6}\left(\ln \frac{M_{c}^{2}}{-q_{o}^{2}}-\gamma_{\omega}\right)^{2}+\frac{1}{6} \alpha_{\omega}\right. \\
& \left.\left.-\frac{5}{18}\left(\ln \frac{M_{c}^{2}}{-p^{2}}-\gamma_{\omega}\right)+\frac{11}{9}\left(\ln \frac{M_{c}^{2}}{-q_{o}^{2}}-\gamma_{\omega}\right)\right]\right\}
\end{aligned}
$$

In order to see the cancellation of harmful or nonlocal terms for diagram $\left(a_{1}\right)$, we need to compute its corresponding counterterm diagram $\left(a_{1}^{\prime}\right)$, as shown on the left in Fig. (3), and this diagram contributes
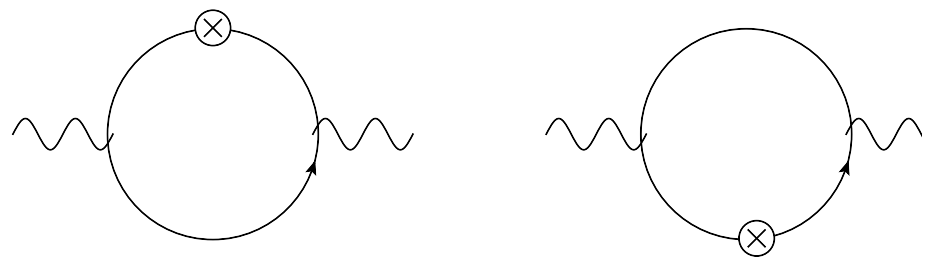

FIG. 3: Counterterm Insertion Diagrams for Diagram Group $(a)$. Left: $\left(a_{1}^{\prime}\right)$; Right: $\left(a_{2}^{\prime}\right)$

$$
\begin{aligned}
\mathcal{M}^{\left(a_{1}^{\prime}\right)} & =\mathcal{M}^{\left(a_{2}^{\prime}\right)}=(-i e)^{2}(-1) \int \frac{d^{4} k}{(2 \pi)^{4}} \operatorname{tr}\left[\gamma^{\mu} \frac{i}{\not p+\not p} \gamma^{\nu} \frac{i}{\not \phi}\left(i \not h \delta_{2}\right) \frac{i}{\not \phi}\right] \\
& =\left(-\delta_{2}\right)(-1)(-i e)^{2} \int \frac{d^{4} k}{(2 \pi)^{4}} \operatorname{tr}\left(\gamma^{\mu} \frac{i}{\not \phi+\not p} \gamma^{\nu} \frac{i}{\not p}\right) \\
& =-\frac{4 i e^{4}}{\left(16 \pi^{2}\right)^{2}}\left(g^{\mu \nu} p^{2}-p^{\mu} p^{\nu}\right)\left[\frac{1}{3}\left(\ln \frac{M_{c}^{2}}{\mu^{2}}-\gamma_{\omega}\right)^{2}\right. \\
& \left.+\frac{1}{3}\left(\ln \frac{M_{c}^{2}}{\mu^{2}}-\gamma_{\omega}\right) \ln \frac{\mu^{2}}{-p^{2}}+\frac{5}{9}\left(\ln \frac{M_{c}^{2}}{\mu^{2}}-\gamma_{\omega}\right)\right]
\end{aligned}
$$


Here we recognized that the internal momentum integration is essentially the same as the photon vacuum polarization at one-loop order and the result Eq.(17) can be directly applied.

By summing up the diagrams $\left(a_{1}\right)$ and $\left(a_{1}^{\prime}\right)$, we can obtain:

$$
\begin{aligned}
\mathcal{M}^{\left(a_{1}+a_{1}^{\prime}\right)}= & \frac{4 i e^{4}}{\left(16 \pi^{2}\right)^{2}}\left\{g^{\mu \nu} M_{c}^{2}\left(\ln \frac{M_{c}^{2}}{-q_{o}^{2}}-\gamma_{\omega}\right)+g^{\mu \nu} p^{2}\left[\frac{1}{6}\left(\ln \frac{M_{c}^{2}}{-q_{o}^{2}}-\gamma_{\omega}\right)-\frac{5}{36}\right]\right. \\
& +\left(g^{\mu \nu} p^{2}-p^{\mu} p^{\nu}\right)\left[-\frac{1}{6}\left(\ln \frac{M_{c}^{2}}{\mu^{2}}-\gamma_{\omega}\right)^{2}+\frac{7}{18}\left(\ln \frac{M_{c}^{2}}{\mu^{2}}-\gamma_{\omega}\right)+\frac{1}{3} \ln \frac{\mu^{2}}{-p^{2}} \ln \frac{\mu^{2}}{-q_{o}^{2}}\right. \\
& \left.\left.-\frac{1}{6} \ln ^{2} \frac{\mu^{2}}{-q_{o}^{2}}-\frac{5}{18} \ln \frac{\mu^{2}}{-p^{2}}+\frac{11}{9} \ln \frac{\mu^{2}}{-q_{o}^{2}}-\frac{1}{6} \alpha_{\omega}-\frac{17}{12}+\frac{\pi^{2}}{18}\right]\right\}
\end{aligned}
$$

Note that there is still a harmful divergence $\frac{4 i e^{4}}{\left(16 \pi^{2}\right)^{2}} g^{\mu \nu} M_{c}^{2} \ln \left(-q_{o}^{2}\right)$, which also breaks the underlying gauge invariance and its associated Ward identity. From the general argument of gauge invariance, we expect that terms like this should not appear in the final result of the two-loop vacuum polarization. As we will see in the following, the result of diagram (b) contains same term with the opposite sign. Therefore, the addition of $\left(a_{1}\right),\left(a_{2}\right)$ and (b) will eliminate this unwanted term, and recover the gauge invariance and locality of the underlying field theory.

\section{Vertex Correction Insertion Diagram}

Now we compute the more challenging diagram in Fig 4. Following the standard Feynman rules of QED, we can write down the explicit expression $\mathcal{M}^{(b)}$

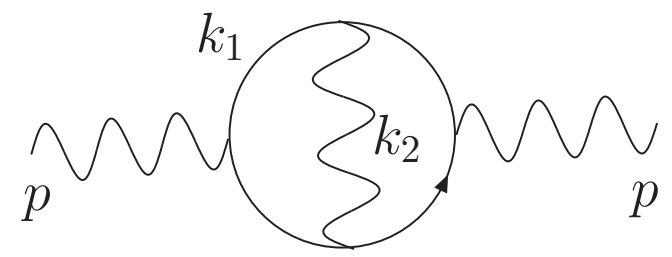

FIG. 4: Vertex Correction Insertion Diagram (b)

$$
\begin{aligned}
& \mathcal{M}^{(b)}=(-i e)^{4} \int \frac{d^{4} k_{2}}{(2 \pi)^{4}} \int \frac{d^{4} k_{1}}{(2 \pi)^{4}}(-1) \operatorname{tr}\left(\gamma^{\mu} \frac{i}{\not \phi_{1}} \gamma^{\rho} \frac{i}{\not \phi_{1}+\not \not_{2}} \gamma^{\nu} \frac{i}{\not \phi_{1}+\not \phi_{2}+\not p} \gamma^{\sigma} \frac{i}{\not \phi_{1}+\not p}\right) \frac{-i g_{\mu \nu}}{k_{2}^{2}} \text {. } \\
& =i e^{4} \int \frac{d^{4} k_{2}}{(2 \pi)^{4}} \int \frac{d^{4} k_{1}}{(2 \pi)^{4}} \int_{0}^{1} d x_{1} \int_{0}^{1} d x_{2} \\
& \frac{\operatorname{tr}\left[\gamma^{\mu}\left(\not \phi_{1}-x_{1} \not \not\right) \gamma^{\rho}\left(\not \not_{1}+\not \not_{2}-x_{1} \not p\right) \gamma^{\nu}\left(\not \not_{1}+\not \not_{2}+\left(1-x_{1}\right) \not p\right) \gamma_{\rho}\left(\not \phi_{1}+\left(1-x_{1}\right) \not p\right)\right]}{\left(k_{1}^{2}+x_{1}\left(1-x_{1}\right) p^{2}\right)^{2}\left[\left(k_{1}+k_{2}+\left(x_{2}-x_{1}\right) p\right)^{2}+x_{2}\left(1-x_{2}\right) p^{2}\right]^{2} k_{2}^{2}}
\end{aligned}
$$

where we have used Feynman parameters to combine some of the denominators. Next, in order to integrate over momentum $k_{1}$, we apply the UVDP parametrization to combine the factors involving $k_{1}$ in the denominator to get

$$
\begin{aligned}
& \frac{1}{\left(k_{1}^{2}+x_{1}\left(1-x_{1}\right) p^{2}\right)^{2}\left[\left(k_{1}+k_{2}+\left(x_{2}-x_{1}\right) p\right)^{2}+x_{2}\left(1-x_{2}\right) p^{2}\right]^{2} k_{2}^{2}} \\
= & \Gamma(4) \int_{0}^{\infty} \prod_{i=i}^{2} \frac{d v_{i}}{\left(1+v_{i}\right)^{2}} \frac{\delta\left(1-\sum_{i=1}^{2} \frac{1}{1+v_{i}}\right) \frac{1}{\left(1+v_{1}\right)\left(1+v_{2}\right)}}{\mathcal{D}^{4} k_{2}^{2}}
\end{aligned}
$$


where we define

$$
\begin{aligned}
\mathcal{D} \equiv & \frac{1}{1+v_{1}}\left[k_{1}^{2}+x_{1}\left(1-x_{1}\right) p^{2}\right]+\frac{1}{1+v_{2}}\left\{\left[k_{1}+k_{2}+\left(x_{2}-x_{1}\right) p\right]^{2}+x_{2}\left(1-x_{2}\right) p^{2}\right\} \\
= & \left(k_{1}+\frac{1}{1+v_{2}} k_{2}+\frac{x_{2}-x_{1}}{1+v_{2}} p\right)^{2} \\
& +\frac{1}{\left(1+v_{1}\right)\left(1+v_{2}\right)}\left[k_{2}+\left(x_{2}-x_{1}\right) p\right]^{2}+\left[\frac{x_{1}\left(1-x_{1}\right)}{1+v_{1}}+\frac{x_{2}\left(1-x_{2}\right)}{1+v_{2}}\right] p^{2} \\
\equiv & \left(k_{1}+\frac{1}{1+v_{2}} k_{2}+\frac{x_{2}-x_{1}}{1+v_{2}} p\right)^{2}+M_{k_{2}, p}^{2} .
\end{aligned}
$$

Then we make the following translation

$$
k_{1} \longrightarrow k_{1}-\frac{1}{1+v_{2}} k_{2}-\frac{x_{2}-x_{1}}{1+v_{2}} p
$$

By expanding the trace of the product of gamma matrices and contracting the Lorentz indices, we write the numerator as,

$$
\begin{aligned}
\mathcal{N}= & \operatorname{tr}\left[\gamma^{\mu}\left(\not k_{1}+l_{1}\right) \gamma^{\rho}\left(\not l_{1}+l_{2}\right) \gamma^{\nu}\left(\not k_{1}+l_{3}\right) \gamma_{\rho}\left(\not k_{1}+l_{4}\right)\right] \\
= & (-8)\left\{g^{\mu \nu} k_{1}^{4}+\left[2 k_{1}^{\mu} k_{1}^{\nu} l_{3} \cdot l_{4}-g^{\mu \nu} k_{1}^{2} l_{3} \cdot l_{4}-2 k_{1}^{\mu} l_{4}^{\nu} l_{3} \cdot k_{1}-2 l_{3}^{\mu} k_{1}^{\nu} l_{4} \cdot k_{1}\right.\right. \\
& \left.+2 g^{\mu \nu} l_{3} \cdot k_{1} l_{4} \cdot k_{1}+l_{4}^{\mu} l_{3}^{\nu} k_{1}^{2}+l_{3}^{\mu} l_{4}^{\nu} k_{1}^{2}\right]+\left[l_{4}^{\mu} l_{2}^{\nu} k_{1}^{2}-l_{2}^{\mu} l_{4}^{\nu} k_{1}^{2}+g^{\mu \nu} l_{2} \cdot l_{4} k_{1}^{2}\right] \\
& +\left[-2 k_{1}^{\mu} k_{1}^{\nu} l_{2} \cdot l_{3}+g^{\mu \nu} k_{1}^{2} l_{2} \cdot l_{3}+2 k_{1}^{\mu} l_{3}^{\nu} l_{2} \cdot k_{1}+2 k_{1}^{\mu} l_{2}^{\nu} l_{3} \cdot k_{1}-l_{3}^{\mu} l_{2}^{\nu} k_{1}^{2}-l_{2}^{\mu} l_{3}^{\nu} k_{1}^{2}\right] \\
& +\left[-l_{3}^{\mu} l_{1}^{\nu} k_{1}^{2}+l_{1}^{\mu} l_{3}^{\nu} k_{1}^{2}+g^{\mu \nu} l_{1} \cdot l_{3} k_{1}^{2}\right]+\left[-2 k_{1}^{\mu} k_{1}^{\nu} l_{1} \cdot l_{4}+g^{\mu \nu} k_{1}^{2} l_{1} \cdot l_{4}+2 l_{4}^{\mu} k_{1}^{\nu} l_{1} \cdot k_{1}\right. \\
& \left.+2 l_{1}^{\mu} k_{1}^{\nu} l_{4} \cdot k_{1}-l_{4}^{\mu} l_{1}^{\nu} k_{1}^{2}-l_{1}^{\mu} l_{4}^{\mu} k_{1}^{2}\right]+\left[2 k_{1}^{\mu} k_{1}^{\nu} l_{1} \cdot l_{2}-g^{\mu \nu} k_{1}^{2} l_{1} \cdot l_{2}-2 l_{2}^{\mu} k_{1}^{\nu} l_{1} \cdot k_{1}\right. \\
& \left.\left.-2 k_{1}^{\mu} l_{1}^{\nu} l_{2} \cdot k_{1}+2 g^{\mu \nu} l_{1} \cdot k_{1} l_{2} \cdot k_{1}+l_{1}^{\mu} l_{2}^{\nu} k_{1}^{2}+l_{2}^{\mu} l_{1}^{\nu} k_{1}^{2}\right]\right\}+L_{1234}^{\mu \nu},
\end{aligned}
$$

where $L_{1234}^{\mu \nu} \equiv \operatorname{tr}\left[\gamma^{\mu} l_{1} \gamma^{\rho} l_{2} \gamma^{\nu} l_{3} \gamma_{\rho} l_{4}\right]$. Note that in above expression, we have used $l_{i}$ s to simplify our notation which represent the four factors in the Eq.(25) after the translation of Eq.(28). Due to their complexity, we do not write them out explicitly.

After the manipulation above, the integration over the loop momentum $k_{1}$ is straightforward,

$$
\begin{aligned}
\mathcal{M}^{(b)}= & \frac{8 e^{4} \Gamma(4)}{16 \pi^{2}} \int_{0}^{1} \prod_{i=1}^{2} d x_{i} \int_{0}^{\infty} \prod_{i=1}^{2} \frac{d v_{i}}{\left(1+v_{i}\right)^{3}} \delta\left(1-\sum_{i=1}^{2} \frac{1}{1+v_{i}}\right) \int \frac{d^{4} k_{2}}{(2 \pi)^{4}} \frac{1}{k_{2}^{2}}\left\{g^{\mu \nu}\left(16 \pi^{2} I_{0}^{R}-\frac{5}{6}\right)\right. \\
& +\frac{1}{3 M_{k_{2}, p}^{2}} l_{4}^{\mu} l_{3}^{\nu}+\frac{1}{3 M_{k_{2}, p}^{2}}\left(l_{4}^{\mu} l_{2}^{\nu}-l_{2}^{\mu} l_{4}^{\nu}+g^{\mu \nu} l_{2} \cdot l_{4}\right)+\frac{1}{6 M_{k_{2}, p}^{2}}\left(g^{\mu \nu} l_{2} \cdot l_{3}-l_{2}^{\mu} l_{3}^{\nu}-l_{3}^{\mu} l_{2}^{\nu}\right) \\
& +\frac{1}{3 M_{k_{2}, p}^{2}}\left(l_{1}^{\mu} l_{3}^{\nu}-l_{3}^{\mu} l_{1}^{\nu}+g^{\mu \nu} l_{1} \cdot l_{3}\right)+\frac{1}{6 M_{k_{2}, p}^{2}}\left(g^{\mu \nu} l_{1} \cdot l_{4}-l_{1}^{\mu} l_{4}^{\nu}-l_{4}^{\mu} l_{1}^{\nu}\right) \\
& \left.+\frac{1}{3 M_{k_{2}, p}^{2}} l_{1}^{\mu} l_{2}^{\nu}+\frac{1}{6 M_{k_{2}, p}^{4}} L_{1234}^{\mu \nu}\right\} .
\end{aligned}
$$

Now we integrate over the loop momentum $k_{2}$. By using the UVDP parametrization, we 
combine the two factors containing $k_{2}^{2}$ in the denominators

$$
\begin{aligned}
\frac{1}{k_{2}^{2} M_{k_{2}, p}^{2}} & =\int_{0}^{\infty} \frac{d u}{(1+u)^{2}} \frac{1}{\left[\left(1-\frac{1}{1+u}\right) k_{2}^{2}+\frac{1}{1+u} M_{k_{2}, p}^{2}\right]^{2}} \\
& =\int_{0}^{\infty} d u \frac{1}{\left(u k_{2}^{2}+M_{k_{2}, p}^{2}\right)^{2}} \\
& =\int_{0}^{\infty} d u \frac{1}{\left[u+\frac{1}{\left(1+v_{1}\right)\left(1+v_{2}\right)}\right]^{2}} \frac{1}{\left[\left(k_{2}+\frac{x_{2}-x_{1}}{1+u\left(1+v_{1}\right)\left(1+v_{2}\right)} p\right)^{2}-\mu_{u}^{2}\right]^{2}} \\
\frac{1}{k_{2}^{2} M_{k_{2}, p}^{4}} & =2 \int_{0}^{\infty} d u \frac{1}{\left[u+\frac{1}{\left(1+v_{1}\right)\left(1+v_{2}\right)}\right]^{3}} \frac{1}{\left[\left(k_{2}+\frac{x_{2}-x_{1}}{1+u\left(1+v_{1}\right)\left(1+v_{2}\right)} p\right)^{2}-\mu_{u}^{2}\right]^{3}} \\
\frac{1}{k_{2}^{2}} I_{0}^{R}= & \int \frac{\left[d^{4} k_{1}\right]_{l}}{(2 \pi)^{4}} \frac{1}{k_{2}^{2}\left(k_{1}^{2}-M_{k_{2}, p}^{2}\right)^{2}} \\
= & -\int^{\left[d^{4} k_{1}\right]_{l}} \frac{\Gamma(3)}{(2 \pi)^{4}} \frac{1}{\Gamma(2) \Gamma(1)} \int_{0}^{\infty} d u \frac{1}{\left[k_{1}^{2}-u k_{2}^{2}-M_{k_{2}, p}^{2}\right]^{3}} \\
= & \frac{1}{16 \pi^{2}} \int_{0}^{\infty} d u \frac{1}{u+\frac{1}{\left(1+v_{1}\right)\left(1+v_{2}\right)}} \frac{1}{\left(k_{2}+\frac{x_{2}-x_{1}}{1+u\left(1+v_{1}\right)\left(1+v_{2}\right)} p\right)^{2}-\mu_{u}^{2}}
\end{aligned}
$$

where we have defined

$$
\begin{aligned}
\mu_{u}^{2} \equiv & \left\{\frac{1}{u+\frac{1}{\left(1+v_{1}\right)\left(1+v_{2}\right)}}\left[\frac{\left(x_{2}-x_{1}\right)^{2}}{\left(1+v_{1}\right)\left(1+v_{2}\right)}+\frac{x_{1}\left(1-x_{1}\right)}{1+v_{1}}+\frac{x_{2}\left(1-x_{2}\right)}{1+v_{2}}\right]\right. \\
& \left.-\frac{1}{\left[u+\frac{1}{\left(1+v_{1}\right)\left(1+v_{2}\right)}\right]^{2}} \frac{\left(x_{2}-x_{1}\right)^{2}}{\left(1+v_{1}\right)^{2}\left(1+v_{2}\right)^{2}}\right\}\left(-p^{2}\right) .
\end{aligned}
$$

After performing Wick rotation of $k_{2}$, we can integrate out $k_{2}$ with the LORE method, and this naturally separates the $\mathcal{M}^{(b)}$ into four parts according to the different powers of the factor $\frac{1}{u+\frac{1}{\left(1+v_{1}\right)\left(1+v_{2}\right)}}$. The resulting expression is lengthy, so in order to keep the paper in a readable length, we do not write it here. Appendix C gives the explicit form for each part, as well as the careful calculation of the remaining UVDP parameter integrations. We recommend the interested reader to resort to the Appendix $\mathrm{C}$ for such details. Below we only present our final results for the vertex correction insertion diagram $(b)$ :

$$
\begin{aligned}
\mathcal{M}^{(b)} \sim & -\frac{8 i e^{4}}{\left(16 \pi^{2}\right)^{2}}\left\{g^{\mu \nu} M_{c}^{2}\left(\ln \frac{M_{c}^{2}}{-q_{o}^{2}}-\gamma_{\omega}\right)+\frac{1}{6} g^{\mu \nu} p^{2}\left(\ln \frac{M_{c}^{2}}{-q_{o}^{2}}-\gamma_{\omega}\right)\right. \\
& +\left(g^{\mu \nu} p^{2}-p^{\mu} p^{\nu}\right) \cdot\left[\frac{1}{3}\left(\ln \frac{M_{c}^{2}}{-p^{2}}-\gamma_{\omega}\right)\left(\ln \frac{M_{c}^{2}}{-q_{o}^{2}}-\gamma_{\omega}\right)+\frac{19}{18}\left(\ln \frac{M_{c}^{2}}{-p^{2}}-\gamma_{\omega}\right)\right] \\
& \left.-\frac{1}{3}\left(\ln \frac{M_{c}^{2}}{-q_{o}^{2}}-\gamma_{\omega}\right)+\frac{1}{3}\left[\frac{13}{6}\left(\ln \frac{M_{c}^{2}}{-q_{o}^{2}(1+V)}-\gamma_{\omega}\right)-\frac{1}{2}\left(\ln \frac{M_{c}^{2}}{-q_{o}^{2}}-\gamma_{\omega}\right)^{2}+\frac{1}{2} \alpha_{\omega}\right]\right\} .
\end{aligned}
$$

The counterterm diagrams $\left(b_{1}^{\prime}\right)$ and $\left(b_{2}^{\prime}\right)$ for $(b)$ can be computed directly and the result is

$$
\begin{aligned}
\mathcal{M}^{\left(b_{1}^{\prime}\right)} & =\mathcal{M}^{\left(b_{2}^{\prime}\right)}=(-1)(-i e)^{2} \int \frac{d^{4} k}{(2 \pi)^{4}} \operatorname{tr}\left[\left(\gamma^{\mu} \delta_{1}\right) \frac{i}{\not \phi+\not p} \gamma^{\nu} \frac{i}{\not \phi}\right] \\
& =\frac{4 i e^{4}}{\left(16 \pi^{2}\right)^{2}}\left(g^{\mu \nu} p^{2}-p^{\mu} p^{\nu}\right)\left[\frac{1}{3}\left(\ln \frac{M_{c}^{2}}{\mu^{2}}-\gamma_{\omega}\right)^{2}+\frac{1}{3}\left(\ln \frac{M_{c}^{2}}{\mu^{2}}-\gamma_{\omega}\right) \ln \frac{\mu^{2}}{-p^{2}}+\frac{5}{9}\left(\ln \frac{M_{c}^{2}}{\mu^{2}}-\gamma_{\omega}\right)\right],
\end{aligned}
$$



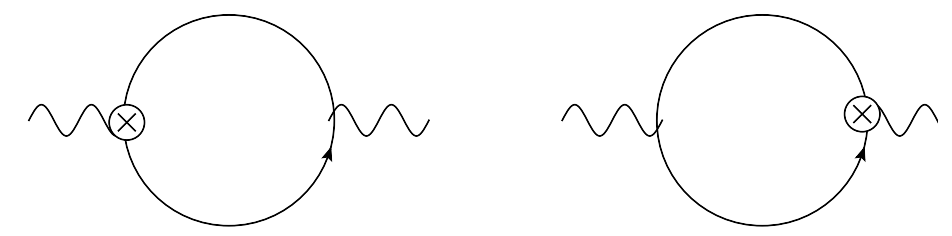

FIG. 5: Counterterm Insertion Diagrams for Diagram (b). Left: $\left(b_{1}^{\prime}\right)$; Right: $\left(b_{2}^{\prime}\right)$

and the summation of $(b),\left(b_{1}^{\prime}\right)$ and $\left(b_{2}^{\prime}\right)$ gives:

$$
\begin{aligned}
\mathcal{M}^{\left(b+b_{1}^{\prime}+b_{2}^{\prime}\right)} \sim & -\frac{8 i e^{4}}{\left(16 \pi^{2}\right)^{2}}\left\{g^{\mu \nu} M_{c}^{2}\left(\ln \frac{M_{c}^{2}}{-q_{o}^{2}}-\gamma_{\omega}\right)+\frac{1}{6} g^{\mu \nu} p^{2}\left(\ln \frac{M_{c}^{2}}{-q_{o}^{2}}-\gamma_{\omega}\right)\right. \\
& +\left(g^{\mu \nu} p^{2}-p^{\mu} p^{\nu}\right) \cdot\left[-\frac{1}{6}\left(\ln \frac{M_{c}^{2}}{\mu^{2}}-\gamma_{\omega}\right)^{2}+\frac{8}{9}\left(\ln \frac{M_{c}^{2}}{\mu^{2}}-\gamma_{\omega}\right)+\frac{1}{3} \ln \frac{\mu^{2}}{-p^{2}} \ln \frac{\mu^{2}}{-q_{o}^{2}}\right. \\
& \left.\left.-\frac{1}{6} \ln ^{2} \frac{\mu^{2}}{-q_{o}^{2}}+\frac{19}{18} \ln \frac{\mu^{2}}{-p^{2}}+\frac{7}{18} \ln \frac{\mu^{2}}{-q_{o}^{2}}-\frac{1}{6} \alpha_{\omega}\right]\right\}
\end{aligned}
$$

As expected from the argument in the end of the subsection IIIB, the results of summing over the diagrams $\left(b+b_{1}^{\prime}+b_{2}^{\prime}\right)$ also possess a harmful divergence which violates the gaugeinvariance. As we will see, this term has the right value to cancel the harmful divergence in the diagram group $(a)$.

\section{The Results}

With the results Eqs.(24) and (35) for the diagrams $\left(a_{1}+a_{1}^{\prime}\right)$ and $\left(b+b_{1}^{\prime}+b_{2}^{\prime}\right)$ at hands, it is straightforward to obtain the final result of QED vacuum polarization at two-loop order:

$$
\begin{aligned}
\mathcal{M}^{\text {final }}= & 2 \mathcal{M}^{\left(a_{1}+a_{1}^{\prime}\right)}+\mathcal{M}^{\left(b+b_{1}^{\prime}+b_{2}^{\prime}\right)} \\
\sim & \frac{8 i e^{4}}{\left(16 \pi^{2}\right)^{2}}\left\{g^{\mu \nu} M_{c}^{2}\left(\ln \frac{M_{c}^{2}}{-q_{o}^{2}}-\gamma_{\omega}\right)+g^{\mu \nu} p^{2}\left[\frac{1}{6}\left(\ln \frac{M_{c}^{2}}{-q_{o}^{2}}-\gamma_{\omega}\right)-\frac{5}{36}\right]\right. \\
& +\left(g^{\mu \nu} p^{2}-p^{\mu} p^{\nu}\right)\left[-\frac{1}{6}\left(\ln \frac{M_{c}^{2}}{\mu^{2}}-\gamma_{\omega}\right)^{2}+\frac{7}{18}\left(\ln \frac{M_{c}^{2}}{\mu^{2}}-\gamma_{\omega}\right)+\frac{1}{3} \ln \frac{\mu^{2}}{-p^{2}} \ln \frac{\mu^{2}}{-q_{o}^{2}}\right. \\
& \left.\left.-\frac{1}{6} \ln ^{2} \frac{\mu^{2}}{-q_{o}^{2}}-\frac{5}{18} \ln \frac{\mu^{2}}{-p^{2}}+\frac{11}{9} \ln \frac{\mu^{2}}{-q_{o}^{2}}-\frac{1}{6} \alpha_{\omega}-\frac{17}{12}+\frac{\pi^{2}}{18}\right]\right\} \\
& -\frac{8 i e^{4}}{\left(16 \pi^{2}\right)^{2}}\left\{g^{\mu \nu} M_{c}^{2}\left(\ln \frac{M_{c}^{2}}{-q_{o}^{2}}-\gamma_{\omega}\right)+\frac{1}{6} g^{\mu \nu} p^{2}\left(\ln \frac{M_{c}^{2}}{-q_{o}^{2}}-\gamma_{\omega}\right)\right. \\
& +\left(g^{\mu \nu} p^{2}-p^{\mu} p^{\nu}\right) \cdot\left[-\frac{1}{6}\left(\ln \frac{M_{c}^{2}}{\mu^{2}}-\gamma_{\omega}\right)^{2}+\frac{8}{9}\left(\ln \frac{M_{c}^{2}}{\mu^{2}}-\gamma_{\omega}\right)+\frac{1}{3} \ln \frac{\mu^{2}}{-p^{2}} \ln \frac{\mu^{2}}{-q_{o}^{2}}\right. \\
& \left.\left.-\frac{1}{6} \ln ^{2} \frac{\mu^{2}}{-q_{o}^{2}}+\frac{19}{18} \ln \frac{\mu^{2}}{-p^{2}}+\frac{7}{18} \ln \frac{\mu^{2}}{-q_{o}^{2}}-\frac{1}{6} \alpha_{\omega}\right]\right\} \\
\sim & \frac{8 i e^{4}}{\left(16 \pi^{2}\right)^{2}}\left(g^{\mu \nu} p^{2}-p^{\mu} p^{\nu}\right)\left[-\frac{4}{3}\left(\ln \frac{M_{c}^{2}}{-p^{2}}-\gamma_{\omega}\right)+\frac{5}{6}\left(\ln \frac{M_{c}^{2}}{-q_{o}^{2}}-\gamma_{\omega}\right)\right] .
\end{aligned}
$$

Note that there is a free mass scale $-q_{o}^{2}$ in the above expression, which was introduced to transform the divergent UVDP-parameter integrals into ILI-like one and the latter is the 
right object to be regularized in the framework of LORE method. If this scale is left in the final result, we must ask what is the physical meaning of this new scale and what the exact value is. In fact, as argued in our previous paper[1], this scale should be determined in terms of the intrinsic energy scales in the theory, like the external momenta or particle masses. In the present case, the only energy scale is the square of the external photon's momentum $p^{2}$, which, in the massless electron case, needs to be space-like. Thus, if the $-q_{o}^{2}$ has some physical meaning, it must have some relation to $p^{2}$. The simplest possibility is that they are the same, $-q_{o}^{2}=-p^{2}$. By applying the relation $-q_{o}^{2}=-p^{2}$, we can reach our final result of photon vacuum polarization diagrams at two loop order:

$$
\begin{aligned}
\mathcal{M}^{\text {final }} & \sim \frac{8 i e^{4}}{\left(16 \pi^{2}\right)^{2}}\left(g^{\mu \nu} p^{2}-p^{\mu} p^{\nu}\right)\left(-\frac{1}{2}\right)\left(\ln \frac{M_{c}^{2}}{-p^{2}}-\gamma_{\omega}\right) \\
& =\frac{i \alpha^{2}}{4 \pi^{2}}\left(p^{\mu} p^{\nu}-g^{\mu \nu} p^{2}\right)\left(\ln \frac{M_{c}^{2}}{-p^{2}}-\gamma_{\omega}\right),
\end{aligned}
$$

where $\alpha=\frac{e^{2}}{4 \pi^{2}}$ is the fine structure constant.

In the light of our result, we have a few comments:

1. As we have already seen, the quadratic divergences are canceled, and this is crucial to guarantee that photon does not obtain a mass from quantum fluctuation and that the whole theory remains gauge invariance.

2. The harmful divergences like $\log p^{2} \cdot \log M_{c}^{2}$ and $M_{c}^{2} \cdot \log p^{2}$ vanishes also, which is expected as these terms are nonlocal and cannot be eliminated by any counterterms in the original Lagrangian which are local. Especially, the quadratic harmful divergence shows a new divergent structure, which can be expected from simple power counting in each of these diagrams. The nontrivial thing is that for every diagram this term will persist even when we include the corresponding counterterm insertion diagrams which only cancel the logarithmically harmful divergence. Only when we sum over all the relevant diagrams do the quadratic harmful divergences cancel each other, rendering the final result local.

Moreover, as emphasized in the previous subsections, this feature is also expected from gauge invariance and the Ward identity in QED. Note that this quadratically harmful divergence is only proportional to $g^{\mu \nu}$ and can give the photon a mass term, both of which are forbidden by the gauge invariance. We think this is a manifestation of the general phenomenon in gauge theories like QCD that although each diagram contains the gauge-violating quadratic divergences, but in the sum of all the relevant diagrams they cancel. The only difference is that this phenomenon occurs at two-loop order for QED while for QCD it happens at one-loop level already [3, 4]. It seems that it is the gauge invariance that rescues us from the harmful divergence "disaster".

3. We can compare our calculation with the LORE method with those using the dimensional regularization (DR). In the calculation with DR we cannot see such cancellation of quadratic harmful divergences because in DR, the final result comes from expansion around the actual space-time dimension 4 and in such a expansion only the logarithmic divergences can be preserved while the quadratic or more higher divergences are simply omitted. It is the resulting incomplete expression in DR that makes this new divergence structure and its elimination invisible. However, since LORE enables us to calculate the full results in the exactly four dimensions, we can easily overcome this problem faced by DR, allowing the appearance of this nontrivial divergence structure. 
4. The final result Eq.(37) is transverse, which implies that our calculation respects gauge invariance and the corresponding Ward Identity. Therefore, the final divergence can be canceled by a single two-loop local counterterm for photon vacuum polarization.

5. Our result agrees with other authors' results [5, 7, 8].

Therefore we come to our conclusion: LORE method, together with the prescription of consistency conditions, can be safely applied to general gauge theories, like QED, at least, up to two-loop order.

\section{CONCLUSION}

In this paper, we mainly discuss the problem how to deal with the general tensor-type high-loop integrals in the framework of LORE. We show that LORE, together with its consistency conditions for 1-folded ILIs, are enough to regularize the tensor-type integrals properly and can be consistently applied to the general gauge theories. In order to show this conclusion, we use the two-loop massless QED vacuum polarization as the simplest gauge theory example to show the general procedure. The final result is sensible: the two-loop correction is transverse and the harmful divergences and quadratic divergences are naturally canceled, which are required by the locality and the gauge invariance and its associated Ward identity of the underlying QED. Consequently, our result agrees with other authors' well-established one. Therefore, the computation of massless QED vacuum polarization at two-loop order explicitly shows LORE is consistent with the gauge invariance and give us confidence to apply it to other realistic theories, like the standard model in particle physics.

What we want to emphasize is that from our present calculation, we have shown a new divergence structure: either the self-energy insertion diagram $\left(a_{1}\right)$ and $\left(a_{2}\right)$ or the vertex correction insertion diagram $(b)$ may contain gauge non-invariant quadratically harmful divergent term even when combined with their own one-loop counterterm diagrams. However, only when we sum over all diagrams in this order the harmful divergences cancel and the final result recovers the gauge invariance and locality. We argue that this feature is general for gauge theories, because the quadratic divergences which violate the gauge invariance will give the gauge boson mass and should be absent in the physical gauge invariant results. Furthermore, the reason why LORE method, rather than other regularization methods like dimensional regularization, can show this new divergence structure lies in the fact that LORE enables us to calculate the complete expression for any Feynman diagram, especially including the quadratic divergences, which is one of main advantages of LORE.

\section{Acknowledgement}

The authors would like to thank Jianwei Cui, Yibo Yang and Yong Tang for useful discussions. This work was supported in part by the National Science Foundation of China (NSFC) under Grant \#No. 10821504, 10975170 and the Project of Knowledge Innovation Program (PKIP) of the Chinese Academy of Science. 


\section{Appendix A: Useful Formula for the Regularization ILIs in LORE}

In the following, we will list some of the most useful regularized ILIs for reference:

$$
\begin{aligned}
& \int\left[d^{4} k\right]_{l} \frac{1}{k^{2}+M_{l}^{2}+\mathcal{M}^{2}}=\pi^{2}\left\{M_{c}^{2}-\mu^{2}\left[\ln \frac{M_{c}^{2}}{\mu^{2}}-\gamma_{w}+1+y_{2}\left(\frac{\mu^{2}}{M_{c}^{2}}\right)\right]\right\} \\
& \int\left[d^{4} k\right]_{l} \frac{1}{\left(k^{2}+M_{l}^{2}+\mathcal{M}^{2}\right)^{2}}=\pi^{2}\left\{\ln \frac{M_{c}^{2}}{\mu^{2}}-\gamma_{w}+y_{0}\left(\frac{\mu^{2}}{M_{c}^{2}}\right)\right\} \\
& \int\left[d^{4} k\right]_{l} \frac{1}{\left(k^{2}+M_{l}^{2}+\mathcal{M}^{2}\right)^{3}}=\frac{1}{2 \mu^{2}} \pi^{2}\left\{1-y_{-2}\left(\frac{\mu^{2}}{M_{c}^{2}}\right)\right\} \\
& \int\left[d^{4} k\right]_{l} \frac{1}{\left(k^{2}+M_{l}^{2}+\mathcal{M}^{2}\right)^{\alpha}}=\pi^{2} \frac{\Gamma(\alpha-2)}{\Gamma(\alpha)} \frac{1}{\left(\mu^{2}\right)^{\alpha-2}}\left\{1-y_{-2(\alpha-2)}\left(\frac{\mu^{2}}{M_{c}^{2}}\right)\right\} \quad \alpha>3 \\
& \int\left[d^{4} k\right]_{l} \frac{k \mu k_{\nu}}{\left(k^{2}+M_{l}^{2}+\mathcal{M}^{2}\right)^{2}}=\frac{1}{2} \delta_{\mu \nu} \pi^{2}\left\{M_{c}^{2}-\mu^{2}\left[\ln \frac{M_{c}^{2}}{\mu^{2}}-\gamma_{w}+1+y_{2}\left(\frac{\mu^{2}}{M_{c}^{2}}\right)\right]\right\} \\
& \int\left[d^{4} k\right]_{l} \frac{k \mu k_{\nu}}{\left(k^{2}+M_{l}^{2}+\mathcal{M}^{2}\right)^{3}}=\frac{1}{4} \delta_{\mu \nu} \pi^{2}\left\{\ln \frac{M_{c}^{2}}{\mu^{2}}-\gamma_{w}+y_{0}\left(\frac{\mu^{2}}{M_{c}^{2}}\right)\right\} \\
& \int\left[d^{4} k\right]_{l} \frac{k \mu k_{\nu}}{\left(k^{2}+M_{l}^{2}+\mathcal{M}^{2}\right)^{4}}=\frac{1}{4} \delta_{\mu \nu} \frac{1}{3 \mu^{2}} \pi^{2}\left\{1-y_{-2}\left(\frac{\mu^{2}}{M_{c}^{2}}\right)\right\} \\
& \int\left[d^{4} k\right]_{l} \frac{k \mu k_{\nu}}{\left(k^{2}+M_{l}^{2}+\mathcal{M}^{2}\right)^{\alpha+1}}=\frac{1}{2} \delta_{\mu \nu} \pi^{2} \frac{\Gamma(\alpha-2)}{\Gamma(\alpha+1)} \frac{1}{\left(\mu^{2}\right)^{\alpha-2}}\left\{1-y_{-2(\alpha-2)}\left(\frac{\mu^{2}}{M_{c}^{2}}\right)\right\} \\
& \int\left[d^{4} k\right]_{l} \frac{k \mu k_{\nu} k_{\rho} k_{\sigma}}{\left(k^{2}+M_{l}^{2}+\mathcal{M}^{2}\right)^{3}}=\frac{1}{8} \delta_{\{\mu \nu \rho \sigma\}} \pi^{2}\left\{M_{c}^{2}-\mu^{2}\left[\ln \frac{M_{c}^{2}}{\mu^{2}}-\gamma_{w}+1+y_{2}\left(\frac{\mu^{2}}{M_{c}^{2}}\right)\right]\right\} \\
& \int\left[d^{4} k\right]_{l} \frac{k \mu k_{\nu} k_{\rho} k_{\sigma}}{\left(k^{2}+M_{l}^{2}+\mathcal{M}^{2}\right)^{4}}=\frac{1}{24} \delta_{\{\mu \nu \rho \sigma\}} \pi^{2}\left\{\ln \frac{M_{c}^{2}}{\mu^{2}}-\gamma_{w}+y_{0}\left(\frac{\mu^{2}}{M_{c}^{2}}\right)\right\} \\
& \int\left[d^{4} k\right]_{l} \frac{k \mu k_{\nu} k_{\rho} k_{\sigma}}{\left(k^{2}+M_{l}^{2}+\mathcal{M}^{2}\right)^{5}}=\frac{1}{24} \delta_{\{\mu \nu \rho \sigma\}} \frac{1}{4 \mu^{2}} \pi^{2}\left\{1-y_{-2}\left(\frac{\mu^{2}}{M_{c}^{2}}\right)\right\} \\
& \int\left[d^{4} k\right]_{l} \frac{k \mu k_{\nu} k_{\rho} k_{\sigma}}{\left(k^{2}+M_{l}^{2}+\mathcal{M}^{2}\right)^{\alpha+2}}=\frac{1}{4} \delta_{\{\mu \nu \rho \sigma\}} \pi^{2} \frac{\Gamma(\alpha-2)}{\Gamma(\alpha+2)} \frac{1}{\left(\mu^{2}\right)^{\alpha-2}}\left\{1-y_{-2(\alpha-2)}\left(\frac{\mu^{2}}{M_{c}^{2}}\right)\right\}
\end{aligned}
$$

with

$$
\int\left[d^{4} k\right]_{l} \equiv \lim _{N \rightarrow \infty} \sum_{l=0}^{N} c_{l}^{N} \int d^{4} k=\lim _{N \rightarrow \infty} \sum_{l=0}^{N}(-1)^{l} \frac{N !}{(N-l) ! l !} \int d^{4} k
$$


where

$$
\begin{aligned}
& M_{l}^{2}=\mu_{s}^{2}+l M_{R}^{2}, \quad M_{R}^{2}=M_{c}^{2} h_{w}(N) \ln N \\
& \mu^{2}=\mu_{s}^{2}+\mathcal{M}^{2} \\
& \delta_{\{\mu \nu \rho \sigma\}} \equiv \delta_{\mu \nu} \delta_{\rho \sigma}+\delta_{\mu \rho} \delta_{\nu \sigma}+\delta_{\mu \sigma} \delta_{\rho \nu} \\
& y_{-2(\alpha-2)}(x)=-\lim _{N} \sum_{l=1}^{N} c_{l}^{N}\left(\frac{x /\left(l h_{w}(N) \ln N\right)}{1+x /\left(l h_{w}(N) \ln N\right)}\right)^{\alpha-2} \\
& \gamma_{w}=\gamma_{E}=0.5772 \cdots, \quad h_{w}(N \rightarrow \infty)=1
\end{aligned}
$$

The explicit form of $y_{-2(\alpha-2)}(x)$ is already given by Eq.(9)

\section{Appendix B: Details of Calculation of Self-Energy Insertion Diagram}

In this Appendix, we shall present the details of the calculation of the UVDP parameter integrals for the self-energy correction insertion diagram $\left(a_{1}\right)$ shown in Fig.(2)).

With the simplification by setting $\mu_{s}^{2} \rightarrow 0$ and $y_{i}\left(\frac{\mu^{2}}{M_{c}^{2}}\right) \rightarrow 0$ in Eq.(21), we have the following expression for $\mathcal{M}_{2}^{\left(a_{1}\right)}$

$$
\begin{aligned}
\mathcal{M}_{2}^{\left(a_{1}\right)}= & \frac{8 i e^{4} \Gamma(4)}{\left(16 \pi^{2}\right)^{2}} \int_{0}^{\infty} \frac{d u}{1+u} \int_{0}^{1} d x \int_{0}^{\infty} \frac{d v_{1} v_{1}}{\left(1+v_{1}\right)^{3}} \frac{1}{6} g^{\mu \nu} \\
& \left\{M_{c}^{2}+\frac{u x v_{1}}{(1+u)^{2}}\left(1-\frac{x v_{1}}{1+v_{1}}\right) p^{2}\left[\left(\ln \frac{M_{c}^{2}}{-p^{2}}-\gamma_{\omega}+1\right)-\ln \left(\frac{u x v_{1}}{(1+u)^{2}}\left(1-\frac{x v_{1}}{1+v_{1}}\right)\right)\right]\right\} \\
= & \mathcal{M}_{22}^{\left(a_{1}\right)}+\mathcal{M}_{20}^{\left(a_{1}\right)}+\mathcal{M}_{2 R}^{\left(a_{1}\right)} .
\end{aligned}
$$

Here $\mathcal{M}_{22}^{\left(a_{1}\right)}, \mathcal{M}_{20}^{\left(a_{1}\right)}$ and $\mathcal{M}_{2 R}^{\left(a_{1}\right)}$ represent quadratical divergent, logarithmical divergent, and regular part in the $\mathcal{M}_{2}^{\left(a_{1}\right)}$.

$$
\begin{aligned}
\mathcal{M}_{22}^{\left(a_{1}\right)} & =\frac{8 i e^{4} \Gamma(4)}{\left(16 \pi^{2}\right)^{2}} \frac{1}{6} g^{\mu \nu} M_{c}^{2} \int_{0}^{\infty} \frac{d u}{1+u} \int_{0}^{1} d x \int_{0}^{\infty} \frac{d v_{1} v_{1}}{\left(1+v_{1}\right)^{3}} \\
& =\frac{8 i e^{4}}{\left(16 \pi^{2}\right)^{2}} g^{\mu \nu} M_{c}^{2} \frac{1}{2} \int_{0}^{\infty} \frac{d u}{1+u} \\
& =\frac{4 i e^{4}}{\left(16 \pi^{2}\right)^{2}} g^{\mu \nu} M_{c}^{2} \int_{0}^{\infty} \frac{\left[d q_{u}^{2}\right] l}{q_{u}^{2}-q_{o}^{2}} \\
& =\frac{4 i e^{4}}{\left(16 \pi^{2}\right)^{2}} g^{\mu \nu} M_{c}^{2}\left[\ln \frac{M_{c}^{2}}{\mu_{s}^{2}-q_{o}^{2}}-\gamma_{\omega}+y_{0}\left(\frac{\mu_{s}^{2}-q_{o}^{2}}{M_{c}^{2}}\right)\right] \\
& =\frac{4 i e^{4}}{\left(16 \pi^{2}\right)^{2}} g^{\mu \nu} M_{c}^{2}\left[\ln \frac{M_{c}^{2}}{-q_{o}^{2}}-\gamma_{\omega}\right]
\end{aligned}
$$

where in the third line we use the trick that $q_{u}^{2} \equiv-q_{o}^{2} u$, which can effectively transform a UVDP parameter integral into a 1-folded irreducible loop integral(ILI) that is the fundamental object to be regulated in the framework of LORE. In the following, we will frequently 
use this trick without any explanation.

$$
\begin{aligned}
\mathcal{M}_{20}^{\left(a_{1}\right)} & =\frac{8 i e^{4} \Gamma(4)}{\left(16 \pi^{2}\right)^{2}} \frac{1}{6} g^{\mu \nu} p^{2}\left(\ln \frac{M_{c}^{2}}{-p^{2}}-\gamma_{\omega}+1\right) \int_{0}^{\infty} \frac{d u u}{(1+u)^{3}} \int_{0}^{1} d x \int_{0}^{\infty} \frac{d v_{1} v_{1}}{\left(1+v_{1}\right)^{3}} x v_{1}\left(1-\frac{x v_{1}}{1+v_{1}}\right) \\
& =\frac{8 i e^{4}}{\left(16 \pi^{2}\right)^{2}} g^{\mu \nu} p^{2}\left(\ln \frac{M_{c}^{2}}{-p^{2}}-\gamma_{\omega}+1\right) \frac{1}{2} \int_{0}^{\infty} \frac{d v_{1} v_{1}^{2}}{\left(1+v_{1}\right)^{3}}\left[\frac{1}{2}-\frac{v_{1}}{3\left(1+v_{1}\right)}\right] \\
& =\frac{4 i e^{4}}{\left(16 \pi^{2}\right)^{2}} g^{\mu \nu} p^{2}\left(\ln \frac{M_{c}^{2}}{-p^{2}}-\gamma_{\omega}+1\right) \cdot\left(\frac{1}{6} \int_{0}^{\infty} \frac{d v_{1}}{1+v_{1}}-\frac{5}{36}\right) \\
& =\frac{4 i e^{4}}{\left(16 \pi^{2}\right)^{2}} g^{\mu \nu} p^{2}\left(\ln \frac{M_{c}^{2}}{-p^{2}}-\gamma_{\omega}+1\right) \cdot\left(\frac{1}{6} \int_{0}^{\infty} \frac{\left[d q_{1}^{2}\right]_{l}}{q_{1}^{2}-q_{o}^{2}}-\frac{5}{36}\right) \\
& =\frac{4 i e^{4}}{\left(16 \pi^{2}\right)^{2}} g^{\mu \nu} p^{2}\left(\ln \frac{M_{c}^{2}}{-p^{2}}-\gamma_{\omega}+1\right) \cdot\left[\frac{1}{6}\left(\ln \frac{M_{c}^{2}}{-q_{o}^{2}}-\gamma_{\omega}\right)-\frac{5}{36}\right]
\end{aligned}
$$

The integral

$$
\begin{aligned}
\mathcal{M}_{2 R}^{\left(a_{1}\right)}= & \frac{8 i e^{4} \Gamma(4)}{\left(16 \pi^{2}\right)^{2}} \frac{1}{6} g^{\mu \nu}\left(-p^{2}\right) \int_{0}^{\infty} \frac{d u u}{(1+u)^{3}} \int_{0}^{1} d x \int_{0}^{\infty} \frac{d v_{1} v_{1}}{\left(1+v_{1}\right)^{3}} \\
& x v_{1}\left(1-\frac{x v_{1}}{1+v_{1}}\right) \ln \left(\frac{u x v_{1}}{(1+u)^{2}}\left(1-\frac{x v_{1}}{1+v_{1}}\right)\right)
\end{aligned}
$$

is difficult since the complicated form in the logarithm. However, if we only focus on its divergence behavior, we can make a lot of simplification. As discussed in Section 2 in our previous paper [1], the analogy between circuit diagram and Fig. (2) tells us that the divergence appear only in the region $v_{1} \rightarrow \infty$. Therefore, we can set $v_{1}$ in the region $v_{1}>V$, which $V$ is a very large number $V \gg 1$. In this asymptotic region, we have $\frac{v_{1}}{1+v_{1}} \rightarrow 1$ and the above expression simplifies to:

$$
\begin{aligned}
\mathcal{M}_{2 R}^{\left(a_{1}\right)} \sim & \frac{8 i e^{4} \Gamma(4)}{\left(16 \pi^{2}\right)^{2}} \frac{1}{6} g^{\mu \nu}\left(-p^{2}\right) \int_{0}^{\infty} \frac{d u u}{(1+u)^{3}} \int_{0}^{1} d x x(1-x) \int_{V}^{\infty} \frac{d v_{1}}{1+v_{1}} \\
& {\left[\ln \frac{u}{(1+u)^{2}}+\ln (x(1-x))+\ln \left(1+v_{1}\right)\right] } \\
\equiv & \mathcal{M}_{2 R u}^{\left(a_{1}\right)}+\mathcal{M}_{2 R x}^{\left(a_{1}\right)}+\mathcal{M}_{2 R v_{1}}^{\left(a_{1}\right)},
\end{aligned}
$$

where $\mathcal{M}_{2 R u}^{\left(a_{1}\right)}, \mathcal{M}_{2 R x}^{\left(a_{1}\right)}$ and $\mathcal{M}_{2 R v_{1}}^{\left(a_{1}\right)}$ represent the three parts in the second line, respectively, the results of which are given as follows:

$$
\begin{aligned}
\mathcal{M}_{2 R u}^{\left(a_{1}\right)} & =\frac{8 i e^{4}}{\left(16 \pi^{2}\right)^{2}}\left(-g^{\mu \nu} p^{2}\right) \int_{0}^{\infty} \frac{d u u}{(1+u)^{3}} \ln \frac{u}{(1+u)^{2}} \int_{0}^{1} d x x(1-x) \int_{V}^{\infty} \frac{d v_{1}}{1+v_{1}} \\
& =\frac{8 i e^{4}}{\left(16 \pi^{2}\right)^{2}}\left(-g^{\mu \nu} p^{2}\right)(-1) \frac{1}{6} \int_{V}^{\infty} \frac{d v_{1}}{1+v_{1}} \\
& =\frac{4 i e^{4}}{\left(16 \pi^{2}\right)^{2}} \frac{1}{3}\left(g^{\mu \nu} p^{2}\right)\left(\ln \frac{M_{c}^{2}}{-q_{o}^{2}(1+V)}-\gamma_{\omega}\right), \\
\mathcal{M}_{2 R x}^{\left(a_{1}\right)} & =\frac{8 i e^{4}}{\left(16 \pi^{2}\right)^{2}}\left(-g^{\mu \nu} p^{2}\right) \int_{0}^{\infty} \frac{d u u}{(1+u)^{3}} \int_{0}^{1} d x x(1-x) \ln [x(1-x)] \int_{V}^{\infty} \frac{d v_{1}}{1+v_{1}} \\
& =\frac{8 i e^{4}}{\left(16 \pi^{2}\right)^{2}}\left(-g^{\mu \nu} p^{2}\right) \frac{1}{2}\left(-\frac{5}{18}\right) \int_{V}^{\infty} \frac{d v_{1}}{1+v_{1}} \\
& =\frac{4 i e^{4}}{\left(16 \pi^{2}\right)^{2}} \frac{5}{18} g^{\mu \nu} p^{2}\left(\ln \frac{M_{c}^{2}}{-q_{o}^{2}(1+V)}-\gamma_{\omega}\right),
\end{aligned}
$$




$$
\begin{aligned}
\mathcal{M}_{2 R v_{1}}^{\left(a_{1}\right)} & =\frac{8 i e^{4}}{\left(16 \pi^{2}\right)^{2}}\left(-g^{\mu \nu} p^{2}\right) \int_{0}^{\infty} \frac{d u u}{(1+u)^{3}} \int_{0}^{1} d x[x(1-x)] \int_{V}^{\infty} \frac{d v_{1}}{1+v_{1}} \ln \left(1+v_{1}\right) \\
& =\frac{4 i e^{4}}{\left(16 \pi^{2}\right)^{2}} \frac{1}{6}\left(-g^{\mu \nu} p^{2}\right) \int_{V}^{\infty} \frac{d v_{1}}{1+v_{1}} \ln \left(1+v_{1}\right) \\
& =\frac{4 i e^{4}}{\left(16 \pi^{2}\right)^{2}} \frac{1}{6}\left(-g^{\mu \nu} p^{2}\right)\left[\int_{0}^{\infty} \frac{d v_{1}}{1+v_{1}} \ln \left(1+v_{1}\right)-\int_{V}^{\infty} \frac{d v_{1}}{1+v_{1}} \ln \left(1+v_{1}\right)\right]
\end{aligned}
$$

Since we are only interested in the divergence behavior, the last finite term can be neglected due to the complexity of its calculation. Below we only give the regulated result for the first term with LORE method and leave the details of derivation to Appendix D.

$$
\begin{aligned}
\mathcal{M}_{2 R v_{1}}^{\left(a_{1}\right)} & \sim \frac{4 i e^{4}}{\left(16 \pi^{2}\right)^{2}} \frac{1}{6}\left(-g^{\mu \nu} p^{2}\right) \int_{0}^{\infty} \frac{d v_{1}}{1+v_{1}} \ln \left(1+v_{1}\right) \\
& =\frac{4 i e^{4}}{\left(16 \pi^{2}\right)^{2}} \frac{1}{12}\left(-g^{\mu \nu} p^{2}\right)\left[\left(\ln \frac{M_{c}^{2}}{-q_{o}^{2}}-\gamma_{\omega}\right)^{2}-\alpha_{\omega}\right] .
\end{aligned}
$$

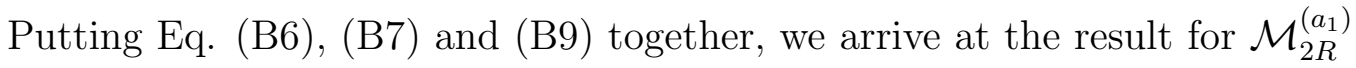

$$
\mathcal{M}_{2 R}^{\left(a_{1}\right)} \sim \frac{4 i e^{4}}{\left(16 \pi^{2}\right)^{2}} g^{\mu \nu} p^{2}\left[\frac{11}{18}\left(\ln \frac{M_{c}^{2}}{-q_{o}^{2}(1+V)}-\gamma_{\omega}\right)-\frac{1}{12}\left(\ln \frac{M_{c}^{2}}{-q_{o}^{2}}-\gamma_{\omega}\right)^{2}+\frac{1}{12} \alpha_{\omega}\right] .
$$

With the experience of the calculation of $\mathcal{M}_{2}^{\left(a_{1}\right)}$, the computation of logarithmic part $\mathcal{M}_{0}^{\left(a_{1}\right)}$ is straightforward:

$$
\begin{aligned}
\mathcal{M}_{0}^{\left(a_{1}\right)}= & \frac{8 i e^{4} \Gamma(4)}{\left(16 \pi^{2}\right)^{2}}\left(-\frac{1}{6}\right)\left(2 p^{\mu} p^{\nu}-g^{\mu \nu} p^{2}\right) \int_{0}^{\infty} \frac{d u u}{(1+u)^{3}} \int_{0}^{1} d x \int_{0}^{\infty} \frac{d v_{1} v_{1}}{\left(1+v_{1}\right)^{3}} x v_{1}\left(1-\frac{x v_{1}}{1+v_{1}}\right) \\
& {\left[\left(\ln \frac{M_{c}^{2}}{-p^{2}}-\gamma_{\omega}\right)-\ln \frac{u x v_{1}}{(1+u)^{2}}\left(1-\frac{x v_{1}}{1+v_{1}}\right)\right] } \\
\equiv & \mathcal{M}_{00}^{\left(a_{1}\right)}+\mathcal{M}_{0 R}^{\left(a_{1}\right)} .
\end{aligned}
$$

¿From the definition of $\mathcal{M}_{00(R)}^{\left(a_{1}\right)}$, it is easy to see that they are essentially the same as counterparts in the quadratic part $\mathcal{M}_{20(R)}^{\left(a_{1}\right)}$. Thus, we can write down the final expression for $\mathcal{M}_{00(R)}^{\left(a_{1}\right)}$ immediately following $\mathcal{M}_{20(R)}^{\left(a_{1}\right)}$.

$$
\begin{gathered}
\mathcal{M}_{00}^{\left(a_{1}\right)}=\frac{4 i e^{4}}{\left(16 \pi^{2}\right)^{2}}\left(g^{\mu \nu} p^{2}-2 p^{\mu} p^{\nu}\right)\left(\ln \frac{M_{c}^{2}}{-p^{2}}-\gamma_{\omega}\right) \cdot\left[\frac{1}{6}\left(\ln \frac{M_{c}^{2}}{-q_{o}^{2}}-\gamma_{\omega}\right)-\frac{5}{36}\right] \\
\mathcal{M}_{0 R}^{\left(a_{1}\right)} \sim \frac{4 i e^{4}}{\left(16 \pi^{2}\right)^{2}}\left(g^{\mu \nu} p^{2}-2 p^{\mu} p^{\nu}\right)\left[\frac{11}{18}\left(\ln \frac{M_{c}^{2}}{-q_{o}^{2}(1+V)}-\gamma_{\omega}\right)-\frac{1}{12}\left(\ln \frac{M_{c}^{2}}{-q_{o}^{2}}-\gamma_{\omega}\right)^{2}+\frac{1}{12} \alpha_{\omega}\right]
\end{gathered}
$$

By summing Eqs. (B2 $),(\overline{\mathrm{B} 3}),(\overline{\mathrm{B} 10}),(\overline{\mathrm{B} 12}),(\overline{\mathrm{B} 13})$, we finally obtain our result for diagram $\left(a_{1}\right)$

$$
\begin{aligned}
\mathcal{M}^{\left(a_{1}\right)} \sim & \frac{4 i e^{4}}{\left(16 \pi^{2}\right)^{2}}\left\{g^{\mu \nu} M_{c}^{2}\left(\ln \frac{M_{c}^{2}}{-q_{o}^{2}}-\gamma_{\omega}\right)+\frac{1}{6} g^{\mu \nu} p^{2}\left(\ln \frac{M_{c}^{2}}{-q_{o}^{2}}-\gamma_{\omega}\right)\right. \\
& +\left(g^{\mu \nu} p^{2}-p^{\mu} p^{\nu}\right) \cdot\left[\frac{1}{3}\left(\ln \frac{M_{c}^{2}}{-p^{2}}-\gamma_{\omega}\right)\left(\ln \frac{M_{c}^{2}}{-q_{o}^{2}}-\gamma_{\omega}\right)-\frac{1}{6}\left(\ln \frac{M_{c}^{2}}{-q_{o}^{2}}-\gamma_{\omega}\right)^{2}+\frac{1}{6} \alpha_{\omega}\right. \\
& \left.\left.-\frac{5}{18}\left(\ln \frac{M_{c}^{2}}{-p^{2}}-\gamma_{\omega}\right)+\frac{11}{9}\left(\ln \frac{M_{c}^{2}}{-q_{o}^{2}}-\gamma_{\omega}\right)\right]\right\}
\end{aligned}
$$




\section{Appendix C: Details of Calculation of Vertex Correction Insertion Diagram}

This appendix gives the details of the calculation of integration of UVDP parameters for the vertex correction insertion diagram $(b)$.

Note that the integration of $k_{2}$ naturally separate $\mathcal{M}^{(b)}$ into four pieces with different powers of factor $\frac{1}{u+\frac{1}{\left(1+v_{1}\right)\left(1+v_{2}\right)}}$, each of which we denote as $\mathcal{M}_{i}^{(b)}, i=0,1,2,3$. In each piece, there are different degree of divergences, quadratically or logarithmically divergent and regular. In the following, we will use a second subscript $2,0, R$ to represent these parts. Let us compute these parts one by one.

Firstly, $\mathcal{M}_{0}^{(b)}$ contains only the single quadratically divergent term $\mathcal{M}_{02}^{(b)}$, whose result can be given directly as:

$$
\begin{aligned}
\mathcal{M}_{02}^{(b)} & =\frac{8 i e^{4} \Gamma(4)}{16 \pi^{2}} \int_{0}^{\infty} \prod_{i=1}^{4} \frac{d v_{i}}{\left(1+v_{i}\right)^{2}} \delta\left(1-\sum_{i=1}^{4} \frac{1}{1+v_{i}}\right) g^{\mu \nu} \frac{5}{6} I_{2}^{R}(0) \\
& =\frac{8 i e^{4} \Gamma(4)}{\left(16 \pi^{2}\right)^{2}} \frac{5}{36} g^{\mu \nu} M_{c}^{2},
\end{aligned}
$$

where, as noted before, we have set $\mu_{s}^{2} \rightarrow 0$ in the end since it can be regarded as just the IR regularization in the LORE and does not affect the UV structure of Feynman integrals. In the following, we will always apply this method to simplify our results.

Also, $\mathcal{M}_{1}^{(b)}$ merely includes one term $\mathcal{M}_{12}^{(b)}$, but the calculation is a little involved since it can be divided further to several pieces $\mathcal{M}_{122}^{(b)}, \mathcal{M}_{120}^{(b)}$ and $\mathcal{M}_{12 R}^{(b)}$ according to the degree of divergences each of them contain:

$$
\begin{aligned}
\mathcal{M}_{12}^{(b)}= & -\frac{8 i e^{4} \Gamma(4)}{16 \pi^{2}} \int_{0}^{1} \prod_{i=1}^{2} d x_{i} \int_{0}^{\infty} \prod_{i=1}^{2} \frac{d v_{i}}{\left(1+v_{i}\right)^{3}} \delta\left(1-\sum_{i=1}^{2} \frac{1}{1+v_{i}}\right) \int_{0}^{\infty} \frac{d u}{u+\frac{1}{\left(1+v_{1}\right)\left(1+v_{2}\right)}} I_{2}^{R} \\
= & -\frac{8 i e^{4} \Gamma(4)}{\left(16 \pi^{2}\right)^{2}} \int_{0}^{1} \prod_{i=1}^{2} d x_{i} \int_{0}^{\infty} \prod_{i=1}^{2} \frac{d v_{i}}{\left(1+v_{i}\right)^{3}} \delta\left(1-\sum_{i=1}^{2} \frac{1}{1+v_{i}}\right) \int_{0}^{\infty} \frac{d u}{u+\frac{1}{\left(1+v_{1}\right)\left(1+v_{2}\right)}} \\
& \left\{M_{c}^{2}-\mu_{u}^{2}\left[\ln \frac{M_{c}^{2}}{\mu_{u}^{2}}-\gamma_{\omega}+1\right]\right\} \\
= & \mathcal{M}_{122}^{(b)}+\mathcal{M}_{120}^{(b)}+\mathcal{M}_{12 R}^{(b)} .
\end{aligned}
$$

Each term above can be calculated straightforwardly:

$$
\begin{aligned}
\mathcal{M}_{122}^{(b)}= & -\frac{8 i e^{4} \Gamma(4)}{\left(16 \pi^{2}\right)^{2}} \int_{0}^{1} \prod_{i=1}^{2} d x_{i} \int_{0}^{\infty} \prod_{i=1}^{2} \frac{d v_{i}}{\left(1+v_{i}\right)^{3}} \delta\left(1-\sum_{i=1}^{2} \frac{1}{1+v_{i}}\right) \int_{0}^{\infty} \frac{d u}{u+\frac{1}{\left(1+v_{1}\right)\left(1+v_{2}\right)}} M_{c}^{2} \\
= & -\frac{8 i e^{4} \Gamma(4)}{\left(16 \pi^{2}\right)^{2}} M_{c}^{2} \int_{0}^{1} \prod_{i=1}^{2} d x_{i} \int_{0}^{\infty} \prod_{i=1}^{2} \frac{d v_{i}}{\left(1+v_{i}\right)^{3}} \delta\left(1-\sum_{i=1}^{2} \frac{1}{1+v_{i}}\right) \int_{0}^{\infty} \frac{d q_{u}^{2}}{q_{u}^{2}-\frac{q_{o}^{2}}{\left(1+v_{1}\right)\left(1+v_{2}\right)}} \\
= & -\frac{8 i e^{4} \Gamma(4)}{\left(16 \pi^{2}\right)^{2}} M_{c}^{2} \int_{0}^{1} \prod_{i=1}^{2} d x_{i} \int_{0}^{\infty} \prod_{i=1}^{2} \frac{d v_{i}}{\left(1+v_{i}\right)^{3}} \delta\left(1-\sum_{i=1}^{2} \frac{1}{1+v_{i}}\right) \\
& {\left[\ln \frac{M_{c}^{2}}{-q_{o}^{2} \frac{1}{\left(1+v_{1}\right)\left(1+v_{2}\right)}}-\gamma_{\omega}\right] } \\
= & -\frac{8 i e^{4} \Gamma(4)}{\left(16 \pi^{2}\right)^{2}} M_{c}^{2}\left[\frac{1}{6}\left(\ln \frac{M_{c}^{2}}{-q_{o}^{2}}-\gamma_{\omega}\right)+\frac{5}{18}\right],
\end{aligned}
$$




$$
\begin{aligned}
\mathcal{M}_{120}^{(b)}= & -\frac{8 i e^{4} \Gamma(4)}{\left(16 \pi^{2}\right)^{2}}\left(\ln \frac{M_{c}^{2}}{-p^{2}}-\gamma_{\omega}\right) \int_{0}^{1} \prod_{i=1}^{2} d x_{i} \int_{0}^{\infty} \prod_{i=1}^{2} \frac{d v_{i}}{\left(1+v_{i}\right)^{3}} \delta\left(1-\sum_{i=1}^{2} \frac{1}{1+v_{i}}\right) \int_{0}^{\infty} d u \\
& \frac{1}{u+\frac{1}{\left(1+v_{1}\right)\left(1+v_{2}\right)}}\left\{\frac{1}{u+\frac{1}{\left(1+v_{1}\right)\left(1+v_{2}\right)}}\left[\frac{\left(x_{2}-x_{1}\right)^{2}}{\left(1+v_{1}\right)\left(1+v_{2}\right)}+\frac{x_{1}\left(1-x_{1}\right)}{1+v_{1}}+\frac{x_{2}\left(1-x_{2}\right)}{1+v_{2}}\right]\right. \\
& \left.-\frac{1}{\left[u+\frac{1}{\left(1+v_{1}\right)\left(1+v_{2}\right)}\right]^{2}} \frac{\left(x_{2}-x_{1}\right)^{2}}{\left(1+v_{1}\right)^{2}\left(1+v_{2}\right)^{2}}\right\} p^{2} \\
= & -\frac{8 i e^{4} \Gamma(4)}{\left(16 \pi^{2}\right)^{2}} p^{2} \frac{13}{72}\left(\ln \frac{M_{c}^{2}}{-p^{2}}-\gamma_{\omega}\right) .
\end{aligned}
$$

Since it is easy to prove that $\mathcal{M}_{12 R}^{(b)}$ is finite and thus irrelevant to our present discussion of UV divergence structure, we omit its calculation here and simply write down the result for $\mathcal{M}_{12}^{(b)}$ as:

$$
\begin{aligned}
\mathcal{M}_{12}^{(b)} & =\mathcal{M}_{122}^{(b)}+\mathcal{M}_{120}^{(b)}+\mathcal{M}_{12 R}^{(b)} \\
& \sim-\frac{8 i e^{4} \Gamma(4)}{\left(16 \pi^{2}\right)^{2}}\left[\frac{1}{6} M_{c}^{2}\left(\ln \frac{M_{c}^{2}}{-q_{o}^{2}}-\gamma_{\omega}\right)+\frac{5}{18} M_{c}^{2}+\frac{13}{72} p^{2}\left(\ln \frac{M_{c}^{2}}{-p^{2}}-\gamma_{\omega}\right)\right],
\end{aligned}
$$

where $\sim$ means that they are equal up to the divergence part.

By observation, $\mathcal{M}_{2}^{(b)}$ contains quadratically and logarithmically divergent parts, $\mathcal{M}_{22}^{(b)}$ and $\mathcal{M}_{20}^{(b)}$. The computation of $\mathcal{M}_{22}^{(b)}$ is much like that of $\mathcal{M}_{12}^{(b)}$ and the result is shown below:

$$
\begin{aligned}
& \mathcal{M}_{22}^{(b)}=-\frac{8 i e^{4} \Gamma(4)}{\left(16 \pi^{2}\right)^{2}} g^{\mu \nu} \int_{0}^{1} \prod_{i=1}^{2} d x_{i} \int_{0}^{\infty} \prod_{i=1}^{2} \frac{d v_{i}}{\left(1+v_{i}\right)^{3}} \delta\left(1-\sum_{i=1}^{2} \frac{1}{1+v_{i}}\right) \int_{0}^{\infty} d u \\
& \frac{-\frac{1}{\left(1+v_{1}\right)\left(1+v_{2}\right)}}{\left[u+\frac{1}{\left(1+v_{1}\right)\left(1+v_{2}\right)}\right]^{2}}\left\{M_{c}^{2}-\mu_{u}^{2}\left[\ln \frac{M_{c}^{2}}{\mu_{u}^{2}}-\gamma_{\omega}+1\right]\right\} \\
& =\mathcal{M}_{222}^{(b)}+\mathcal{M}_{220}^{(b)}+\mathcal{M}_{22 R}^{(b)} \\
& \mathcal{M}_{222}^{(b)}=-\frac{8 i e^{4} \Gamma(4)}{\left(16 \pi^{2}\right)^{2}} g^{\mu \nu} \int_{0}^{1} \prod_{i=1}^{2} d x_{i} \int_{0}^{\infty} \prod_{i=1}^{2} \frac{d v_{i}}{\left(1+v_{i}\right)^{3}} \delta\left(1-\sum_{i=1}^{2} \frac{1}{1+v_{i}}\right) \int_{0}^{\infty} d u \\
& \frac{-\frac{1}{\left(1+v_{1}\right)\left(1+v_{2}\right)}}{\left[u+\frac{1}{\left(1+v_{1}\right)\left(1+v_{2}\right)}\right]^{2}} M_{c}^{2} \\
& =\frac{8 i e^{4} \Gamma(4)}{\left(16 \pi^{2}\right)^{2}} g^{\mu \nu} M_{c}^{2} \\
& \mathcal{M}_{220}^{(b)}=-\frac{8 i e^{4} \Gamma(4)}{\left(16 \pi^{2}\right)^{2}} g^{\mu \nu} \int_{0}^{1} \prod_{i=1}^{2} d x_{i} \int_{0}^{\infty} \prod_{i=1}^{2} \frac{d v_{i}}{\left(1+v_{i}\right)^{3}} \delta\left(1-\sum_{i=1}^{2} \frac{1}{1+v_{i}}\right) \int_{0}^{\infty} d u \\
& \frac{\frac{1}{\left(1+v_{1}\right)\left(1+v_{2}\right)}}{\left[u+\frac{1}{\left(1+v_{1}\right)\left(1+v_{2}\right)}\right]^{2}}\left\{\frac{1}{u+\frac{1}{\left(1+v_{1}\right)\left(1+v_{2}\right)}}\left[\frac{\left(x_{2}-x_{1}\right)^{2}}{\left(1+v_{1}\right)\left(1+v_{2}\right)}+\frac{x_{1}\left(1-x_{1}\right)}{1+v_{1}}+\frac{x_{2}\left(1-x_{2}\right)}{1+v_{2}}\right]\right. \\
& \left.-\frac{1}{\left[u+\frac{1}{\left(1+v_{1}\right)\left(1+v_{2}\right)}\right]^{2}} \frac{\left(x_{2}-x_{1}\right)^{2}}{\left(1+v_{1}\right)^{2}\left(1+v_{2}\right)^{2}}\right\}\left(-p^{2}\right)\left(\ln \frac{M_{c}^{2}}{-p^{2}}-\gamma_{\omega}\right) \\
& =\frac{8 i e^{4} \Gamma(4)}{\left(16 \pi^{2}\right)^{2}} g^{\mu \nu} p^{2} \frac{19}{36 \cdot 6}\left(\ln \frac{M_{c}^{2}}{-p^{2}}-\gamma_{\omega}\right)
\end{aligned}
$$


We can prove that the term $\mathcal{M}_{22 R}^{(b)}$ is finite. So we have the divergence behavior of the integration $\mathcal{M}_{22}^{(b)}$ is:

$$
\mathcal{M}_{22}^{(b)} \sim \frac{8 i e^{4} \Gamma(4)}{\left(16 \pi^{2}\right)^{2}} g^{\mu \nu}\left[\frac{1}{6} M_{c}^{2}+\frac{19}{36 \cdot 6} p^{2}\left(\ln \frac{M_{c}^{2}}{-p^{2}}-\gamma_{\omega}\right)\right]
$$

$\mathcal{M}_{20}^{(b)}$ contains many different Lorentz structures, so it is useful to further refine it into three parts according to their Lorentz structure: $\mathcal{M}_{201}^{(b)}$ represents terms proportional to $p^{\mu} p^{\nu}, \mathcal{M}_{202}^{(b)}$ proportional to $g^{\mu \nu} p^{2}$ and $\mathcal{M}_{203}^{b}$ proportional to $g^{\mu \nu} \mu_{u}^{2}$.

$$
\begin{aligned}
\mathcal{M}_{201}^{(b)}= & -\frac{8 i e^{4} \Gamma(4)}{\left(16 \pi^{2}\right)^{2}} p^{\mu} p^{\nu} \int_{0}^{1} \prod_{i=1}^{2} d x_{i} \int_{0}^{\infty} \prod_{i=1}^{2} \frac{d v_{i}}{\left(1+v_{i}\right)^{3}} \delta\left(1-\sum_{i=1}^{2} \frac{1}{1+v_{i}}\right) \int_{0}^{\infty} d u \\
& \frac{1}{3} \frac{1}{\left[u+\frac{1}{\left(1+v_{1}\right)\left(1+v_{2}\right)}\right]^{2}}\left[\frac{\left(x_{2}-x_{1}\right)^{2}}{\left(1+v_{1}\right)^{2}\left(1+v_{2}\right)^{2}\left[u+\frac{1}{\left(1+v_{1}\right)\left(1+v_{2}\right)}\right]^{2}}-1\right]\left(\ln \frac{M_{c}^{2}}{\mu_{u}^{2}}-\gamma_{\omega}\right) \\
= & \mathcal{M}_{2010}^{(b)}+\mathcal{M}_{201 R}^{(b)},
\end{aligned}
$$

where the last subscript of $\mathcal{M}_{2010(R)}^{(b)}$ represents the divergence degree of the part.

$$
\begin{aligned}
\mathcal{M}_{2010}^{(b)}= & -\frac{8 i e^{4} \Gamma(4)}{\left(16 \pi^{2}\right)^{2}} p^{\mu} p^{\nu} \int_{0}^{1} \prod_{i=1}^{2} d x_{i} \int_{0}^{\infty} \prod_{i=1}^{2} \frac{d v_{i}}{\left(1+v_{i}\right)^{3}} \delta\left(1-\sum_{i=1}^{2} \frac{1}{1+v_{i}}\right) \int_{0}^{\infty} d u \\
& \frac{1}{3} \frac{1}{\left[u+\frac{1}{\left(1+v_{1}\right)\left(1+v_{2}\right)}\right]^{2}}\left[\frac{\left(x_{2}-x_{1}\right)^{2}}{\left(1+v_{1}\right)^{2}\left(1+v_{2}\right)^{2}\left[u+\frac{1}{\left(1+v_{1}\right)\left(1+v_{2}\right)}\right]^{2}}-1\right]\left(\ln \frac{M_{c}^{2}}{-p^{2}}-\gamma_{\omega}\right) \\
= & \frac{8 i e^{4} \Gamma(4)}{\left(16 \pi^{2}\right)^{2}} \frac{17}{54} p^{\mu} p^{\nu}\left(\ln \frac{M_{c}^{2}}{-p^{2}}-\gamma_{\omega}\right) .
\end{aligned}
$$

As before, we can prove that the part $\mathcal{M}_{201 R}^{(b)}$ is finite, so the divergent part for $\mathcal{M}_{201}^{(b)}$ is:

$$
\mathcal{M}_{201}^{(b)} \sim \frac{8 i e^{4} \Gamma(4)}{\left(16 \pi^{2}\right)^{2}} \frac{17}{54} p^{\mu} p^{\nu}\left(\ln \frac{M_{c}^{2}}{-p^{2}}-\gamma_{\omega}\right) .
$$

By the very similar way, we can obtain the result of $\mathcal{M}_{202}^{(b)}$.

$$
\begin{aligned}
\mathcal{M}_{202}^{(b)}= & \frac{8 i e^{4} \Gamma(4)}{\left(16 \pi^{2}\right)^{2}} \cdot \frac{1}{6} g^{\mu \nu} p^{2} \int_{0}^{1} \prod_{i=1}^{2} d x_{i} \int_{0}^{\infty} \prod_{i=1}^{2} \frac{d v_{i}}{\left(1+v_{i}\right)^{3}} \delta\left(1-\sum_{i=1}^{2} \frac{1}{1+v_{i}}\right) \int_{0}^{\infty} d u \\
& \frac{1}{\left[u+\frac{1}{\left(1+v_{1}\right)\left(1+v_{2}\right)}\right]^{2}}\left\{\frac{\left[1-\frac{6}{\left(1+v_{1}\right)\left(1+v_{2}\right)}\right] \frac{\left(x_{2}-x_{1}\right)^{2}}{\left(1+v_{1}\right)^{2}\left(1+v_{2}\right)^{2}}}{\left[u+\frac{1}{\left(1+v_{1}\right)\left(1+v_{2}\right)}\right]^{2}}\right. \\
& +6\left(\frac{x_{1}}{1+v_{1}}+\frac{x_{2}}{1+v_{2}}-\frac{1}{2}\right)\left(\frac{1}{1+v_{1}}-\frac{1}{1+v_{2}}\right) \frac{x_{2}-x_{1}}{\left(1+v_{1}\right)\left(1+v_{2}\right)} \frac{1}{u+\frac{1}{\left(1+v_{1}\right)\left(1+v_{2}\right)}} \\
& \left.-6\left(\frac{x_{1}}{1+v_{1}}+\frac{x_{2}}{1+v_{2}}\right)\left(1-\frac{x_{1}}{1+v_{1}}-\frac{x_{2}}{1+v_{2}}\right)\right\}\left(\ln \frac{M_{c}^{2}}{\mu_{u}^{2}}-\gamma_{\omega}\right) \\
= & \mathcal{M}_{2020}^{(b)}+\mathcal{M}_{202 R}^{(b)}
\end{aligned}
$$


By integrating the expression before $\frac{1}{16 \pi^{2}}\left(\ln \frac{M_{c}^{2}}{-p^{2}}-\gamma_{\omega}\right)$, we obtain $\mathcal{M}_{2020}^{(b)}$

$$
\mathcal{M}_{2020}^{(b)}=-\frac{8 i e^{4} \Gamma(4)}{\left(16 \pi^{2}\right)^{2}} \cdot \frac{5}{24} g^{\mu \nu} p^{2}\left(\ln \frac{M_{c}^{2}}{-p^{2}}-\gamma_{\omega}\right) .
$$

The part of $\mathcal{M}_{202 R}^{(b)}$ can also be proved to be finite, so the divergence part of $\mathcal{M}_{202}^{(b)}$ is:

$$
\mathcal{M}_{202}^{(b)} \sim-\frac{8 i e^{4} \Gamma(4)}{\left(16 \pi^{2}\right)^{2}} \cdot \frac{5}{24} g^{\mu \nu} p^{2}\left(\ln \frac{M_{c}^{2}}{-p^{2}}-\gamma_{\omega}\right)
$$

Much like $\mathcal{M}_{201}^{(b)}$ and $\mathcal{M}_{202}^{(b)}$ in structure, it is expected that the $\mathcal{M}_{203}^{(b)}$ can be calculated similarly. However, a very important new feature will appear: the overlapping divergence structure hidden in our current LORE procedure.

$$
\begin{aligned}
\mathcal{M}_{203}^{(b)}= & \frac{8 i e^{4} \Gamma(4)}{\left(16 \pi^{2}\right)^{2}} g^{\mu \nu} p^{2} \int_{0}^{1} \prod_{i=1}^{2} d x_{i} \int_{0}^{\infty} \prod_{i=1}^{2} \frac{d v_{i}}{\left(1+v_{i}\right)^{3}} \delta\left(1-\sum_{i=1}^{2} \frac{1}{1+v_{i}}\right) \int_{0}^{\infty} d u \\
& \frac{1}{\left[u+\frac{1}{\left(1+v_{1}\right)\left(1+v_{2}\right)}\right]^{2}}\left[-\frac{1}{6}+\frac{1}{\left(1+v_{1}\right)\left(1+v_{2}\right)}\right] \cdot \\
& \left\{\frac{1}{u+\frac{1}{\left(1+v_{1}\right)\left(1+v_{2}\right)}}\left[\frac{\left(x_{2}-x_{1}\right)^{2}}{\left(1+v_{1}\right)\left(1+v_{2}\right)}+\frac{x_{1}\left(1-x_{1}\right)}{1+v_{1}}+\frac{x_{2}\left(1-x_{2}\right)}{1+v_{2}}\right]\right. \\
& \left.-\frac{1}{\left[u+\frac{1}{\left(1+v_{1}\right)\left(1+v_{2}\right)}\right]^{2}} \frac{\left(x_{2}-x_{1}\right)^{2}}{\left(1+v_{1}\right)^{2}\left(1+v_{2}\right)^{2}}\right\} \cdot \\
& \left(\left[\ln \frac{\left.M_{c}^{2}-\gamma_{\omega}\right]-}{-p^{2}}\right]\right. \\
& \ln \left\{\frac{1}{u+\frac{1}{\left(1+v_{1}\right)\left(1+v_{2}\right)}}\left[\frac{\left(x_{2}-x_{1}\right)^{2}}{\left(1+v_{1}\right)\left(1+v_{2}\right)}+\frac{x_{1}\left(1-x_{1}\right)}{1+v_{1}}+\frac{x_{2}\left(1-x_{2}\right)}{1+v_{2}}\right]\right. \\
& \left.-\frac{\left(x_{2}-x_{1}\right)^{2}}{\left[u+\frac{1}{\left(1+v_{1}\right)\left(1+v_{2}\right)}\right]^{2}} \frac{\left(1+v_{1}\right)^{2}\left(1+v_{2}\right)^{2}}{\left(1+\mathcal{M}_{203 R}\right.}\right) \\
= & \mathcal{M}_{2030}^{(b)+\mathcal{M}_{2}^{(b)}}
\end{aligned}
$$

where, $\mathcal{M}_{2030}^{(b)}$ represents the part proportional to $\left(\ln \frac{M_{c}^{2}}{-p^{2}}-\gamma_{\omega}\right)$ while $\mathcal{M}_{203 R}^{(b)}$ for the rest 
regular terms. The integration of $\mathcal{M}_{2030}^{(b)}$ is straightforward.

$$
\begin{aligned}
\mathcal{M}_{2030}^{(b)}= & \frac{8 i e^{4} \Gamma(4)}{\left(16 \pi^{2}\right)^{2}} g^{\mu \nu} p^{2} \int_{0}^{1} \prod_{i=1}^{2} d x_{i} \int_{0}^{\infty} \prod_{i=1}^{2} \frac{d v_{i}}{\left(1+v_{i}\right)^{3}} \delta\left(1-\sum_{i=1}^{2} \frac{1}{1+v_{i}}\right) \int_{0}^{\infty} d u \\
& \frac{1}{\left[u+\frac{1}{\left(1+v_{1}\right)\left(1+v_{2}\right)}\right)^{2}} g^{\mu \nu}\left[-\frac{1}{6}+\frac{1}{\left(1+v_{1}\right)\left(1+v_{2}\right)}\right]\left(\ln \frac{M_{c}^{2}}{-p^{2}}-\gamma_{\omega}\right) \\
& \left\{\frac{1}{u+\frac{1}{\left(1+v_{1}\right)\left(1+v_{2}\right)}}\left[\frac{\left(x_{2}-x_{1}\right)^{2}}{\left(1+v_{1}\right)\left(1+v_{2}\right)}+\frac{x_{1}\left(1-x_{1}\right)}{1+v_{1}}+\frac{x_{2}\left(1-x_{2}\right)}{1+v_{2}}\right]\right. \\
& \left.-\frac{1}{\left[u+\frac{1}{\left(1+v_{1}\right)\left(1+v_{2}\right)}\right]^{2}} \frac{\left(x_{2}-x_{1}\right)^{2}}{\left(1+v_{1}\right)^{2}\left(1+v_{2}\right)^{2}}\right\} \\
= & \frac{8 i e^{4} \Gamma(4)}{\left(16 \pi^{2}\right)^{2}} g^{\mu \nu} p^{2}\left(\ln \frac{M_{c}^{2}}{-p^{2}}-\gamma_{\omega}\right)\left[\frac{1}{12}-\frac{1}{72}\left(\int_{0}^{\infty} \frac{d v_{1}}{1+v_{1}}+\int_{0}^{\infty} \frac{d v_{2}}{1+v_{2}}\right)\right] \\
= & \frac{8 i e^{4} \Gamma(4)}{\left(16 \pi^{2}\right)^{2}} g^{\mu \nu} p^{2}\left(\ln \frac{M_{c}^{2}}{-p^{2}}-\gamma_{\omega}\right)\left[\frac{1}{12}-\frac{1}{36}\left(\ln \frac{M_{c}^{2}}{-q_{o}^{2}}-\gamma_{\omega}\right)\right] .
\end{aligned}
$$

Note that the above parameter integration before $\left(\ln \frac{M_{c}^{2}}{-p^{2}}-\gamma_{\omega}\right)$ is also divergent, which is the signal of overlapping divergences. It is clearer when we use the analogy between the Feynman diagrams and the electric circuits, which tells us that the integration of $v_{1}$ and $v_{2}$ should reproduce the divergences coming from the subdiagrams of left and right vertex corrections in Fig.(44) since $\frac{1}{1+v_{1}}\left(\frac{1}{1+v_{2}}\right)$ times the effective propagator $k_{1}^{2}+x_{1}(1-$ $\left.x_{1}\right) p^{2}\left(\left[\left(k_{1}+k_{2}+\left(x_{2}-x_{1}\right) p\right)\right]^{2}+x_{2}\left(1-x_{2}\right) p^{2}\right)$ for the left(right) circle in Eq.(26). When $v_{1}\left(v_{2}\right)$ tends to infinity, the left effective propagator $\frac{1}{1+v_{1}}\left[k_{1}^{2}+x_{1}\left(1-x_{1}\right) p^{2}\right]$ (the right counterpart $\left.\frac{1}{1+v_{2}}\left\{\left[\left(k_{1}+k_{2}+\left(x_{2}-x_{1}\right) p\right)\right]^{2}+x_{2}\left(1-x_{2}\right) p^{2}\right\}\right)$ approaches zero, which means that the left(right) half circle collapse to a point and that sub-circuit is short-cut in the electric circuit language. This singular behavior will become manifest as the divergence in the final integration of UVDP parameter $v_{1}\left(v_{2}\right)$, just as the ones shown in Eq.(C17) above. Thus, the result in Eq.(C17) can be understood as the subdivergences coming from left and right vertex correction times the overall one $\left(\ln \frac{M_{c}^{2}}{-p^{2}}-\gamma_{\omega}\right)$, which is the definition of overlapping divergence [1, [6].

This overlapping divergence take us some further difficulties that the integration of $\mathcal{M}_{203 R}^{(b)}$ turn out to be divergent, which gives us further contributions to our UV-divergence structure. 


$$
\begin{aligned}
\mathcal{M}_{203 R}^{(b)}= & -\frac{8 i e^{4} \Gamma(4)}{\left(16 \pi^{2}\right)^{2}} g^{\mu \nu} p^{2} \int_{0}^{1} \prod_{i=1}^{2} d x_{i} \int_{0}^{\infty} \prod_{i=1}^{2} \frac{d v_{i}}{\left(1+v_{i}\right)^{3}} \delta\left(1-\sum_{i=1}^{2} \frac{1}{1+v_{i}}\right) \int_{0}^{\infty} d u \\
& \frac{1}{\left[u+\frac{1}{\left(1+v_{1}\right)\left(1+v_{2}\right)}\right]^{2}}\left[-\frac{1}{6}+\frac{1}{\left(1+v_{1}\right)\left(1+v_{2}\right)}\right] \\
& \left\{\frac{1}{u+\frac{1}{\left(1+v_{1}\right)\left(1+v_{2}\right)}}\left[\frac{\left(x_{2}-x_{1}\right)^{2}}{\left(1+v_{1}\right)\left(1+v_{2}\right)}+\frac{x_{1}\left(1-x_{1}\right)}{1+v_{1}}+\frac{x_{2}\left(1-x_{2}\right)}{1+v_{2}}\right]\right. \\
& \left.-\frac{1}{\left[u+\frac{1}{\left(1+v_{1}\right)\left(1+v_{2}\right)}\right]^{2}} \frac{\left(x_{2}-x_{1}\right)^{2}}{\left(1+v_{1}\right)^{2}\left(1+v_{2}\right)^{2}}\right\} \\
& \ln \left\{\frac{1}{u+\frac{1}{\left(1+v_{1}\right)\left(1+v_{2}\right)}}\left[\frac{\left(x_{2}-x_{1}\right)^{2}}{\left(1+v_{1}\right)\left(1+v_{2}\right)}+\frac{x_{1}\left(1-x_{1}\right)}{1+v_{1}}+\frac{x_{2}\left(1-x_{2}\right)}{1+v_{2}}\right]\right. \\
& \left.-\frac{1}{\left[u+\frac{1}{\left(1+v_{1}\right)\left(1+v_{2}\right)}\right]^{2}} \frac{\left(x_{2}-x_{1}\right)^{2}}{\left(1+v_{1}\right)^{2}\left(1+v_{2}\right)^{2}}\right\} .
\end{aligned}
$$

Because of the complication in the logarithmic function, it is difficult to get a closed analytical expression for this kinds of integration. However, if we only focus the divergence behavior of the integral, then we can use the method introduced in the calculation of $\mathcal{M}_{2 R}^{\left(a_{1}\right)}$ to greatly simplify the integral and to obtain the asymptotical results. To disentangle the overlapping divergences, we need first to know in what parameter space region the divergence happen. From our experience when working $\mathcal{M}_{2030}^{(b)}$, the divergences take place when $v_{1} \rightarrow \infty$ and $v_{2} \rightarrow \infty$

Now we consider the region where $v_{1} \rightarrow \infty$ and $v_{2} \rightarrow 0$. Fist we choose a very large number, say $V$, and set the integration region is only confined in $v_{1} \gg V$. In such a region, $\frac{1}{1+v_{1}}$ are small quantities, so we can expand the expression according to $\frac{1}{1+v_{1}}$. The leading term of $\mathcal{M}_{203 R}^{(b)}$ is,

$$
\begin{aligned}
& \mathcal{M}_{203 R v_{1}}^{(b)} \sim \frac{8 i e^{4} \Gamma(4)}{\left(16 \pi^{2}\right)^{2}} \cdot \frac{1}{6} g^{\mu \nu} p^{2} \int_{0}^{1} \prod_{i=1}^{2} d x_{i} \int_{V}^{\infty} \frac{d v_{1}}{\left(1+v_{1}\right)^{3}} \int_{0}^{\infty} d u \frac{x_{2}\left(1-x_{2}\right)}{\left(u+\frac{1}{1+v_{1}}\right)^{3}} \ln \frac{x_{2}\left(1-x_{2}\right)}{u+\frac{1}{1+v_{1}}} \\
= & \frac{8 i e^{4} \Gamma(4)}{\left(16 \pi^{2}\right)^{2}} \cdot \frac{1}{6} g^{\mu \nu} p^{2}\left\{\int_{0}^{1} d x_{2} x_{2}\left(1-x_{2}\right) \ln \left[x_{2}\left(1-x_{2}\right)\right] \int_{V}^{\infty} \frac{d v_{1}}{\left(1+v_{1}\right)^{3}} \int_{0}^{\infty} d u \frac{1}{\left(u+\frac{1}{1+v_{1}}\right)^{3}}\right. \\
& \left.-\int_{0}^{1} d x_{2} x_{2}\left(1-x_{2}\right) \int_{V}^{\infty} \frac{d v_{1}}{\left(1+v_{1}\right)^{3}} \int_{0}^{\infty} d u \frac{1}{\left(u+\frac{1}{1+v_{1}}\right)^{3}} \ln \left[u+\frac{1}{1+v_{1}}\right]\right\} \\
= & \frac{8 i e^{4} \Gamma(4)}{\left(16 \pi^{2}\right)^{2}} \cdot \frac{1}{6} g^{\mu \nu} p^{2}\left\{\left(-\frac{5}{18}\right) \frac{1}{2} \int_{V}^{\infty} \frac{d v_{1}}{1+v_{1}}-\frac{1}{6}\left[\frac{1}{4} \int_{V}^{\infty} \frac{d v_{1}}{1+v_{1}}-\frac{1}{2} \int_{V}^{\infty} \frac{d v_{1}}{1+v_{1}} \ln \left(1+v_{1}\right)\right]\right\} \\
\sim & -\frac{8 i e^{4} \Gamma(4)}{\left(16 \pi^{2}\right)^{2}} \cdot \frac{1}{72} g^{\mu \nu} p^{2}\left[\frac{13}{6}\left(\ln \frac{M_{c}^{2}}{-q_{o}^{2}(1+V)}-\gamma_{\omega}\right)-\frac{1}{2}\left(\ln \frac{M_{c}^{2}}{-q_{o}^{2}}-\gamma_{\omega}\right)^{2}+\frac{1}{2} \alpha_{\omega}\right] .
\end{aligned}
$$

where in the last line we extended the lower bound of the integration range to 0 as before for convenience.

Since in the other asymptotic region $v_{2} \rightarrow \infty, v_{1} \rightarrow 0$, we can obtain a similar expression except for the exchange of $v_{1} \leftrightarrow v_{2}$ and $x_{2} \leftrightarrow x_{1}$. Thus, we can expect to get the same 
asymptotic result. Therefore, the divergence behavior of $\mathcal{M}_{203 R}^{(b)}$ is:

$$
\mathcal{M}_{203 R}^{(b)} \sim-\frac{8 i e^{4} \Gamma(4)}{\left(16 \pi^{2}\right)^{2}} \cdot \frac{1}{36} g^{\mu \nu} p^{2}\left[\frac{13}{6}\left(\ln \frac{M_{c}^{2}}{-q_{o}^{2}(1+V)}-\gamma_{\omega}\right)-\frac{1}{2}\left(\ln \frac{M_{c}^{2}}{-q_{o}^{2}}-\gamma_{\omega}\right)^{2}+\frac{1}{2} \alpha_{\omega}\right] .(
$$

$\mathcal{M}_{3}^{(b)}$ can be naturally divided into three parts $\mathcal{M}_{32}^{(b)}, \mathcal{M}_{30}^{(b)}$ and $\mathcal{M}_{3 R}^{(b)}$ according to the divergence degree followed by integration of loop momentum $k_{2}$. $\mathcal{M}_{32}^{(b)}$ contains only one term, so the calculation is straightforward:

$$
\begin{aligned}
& \mathcal{M}_{32}^{(b)}=-\frac{8 i e^{4} \Gamma(4)}{\left(16 \pi^{2}\right)^{2}} \cdot \frac{1}{3} g^{\mu \nu} \int_{0}^{1} \prod_{i=1}^{2} d x_{i} \int_{0}^{\infty} \prod_{i=1}^{2} \frac{d v_{i}}{\left(1+v_{i}\right)^{3}} \delta\left(1-\sum_{i=1}^{2} \frac{1}{1+v_{i}}\right) \int_{0}^{\infty} d u \\
& \frac{1}{\left[u+\frac{1}{\left(1+v_{1}\right)\left(1+v_{2}\right)}\right]^{3}} \frac{1}{\left(1+v_{1}\right)^{2}\left(1+v_{2}\right)^{2}}\left[M_{c}^{2}-\mu_{u}^{2}\left(\ln \frac{M_{c}^{2}}{\mu_{u}^{2}}-\gamma_{\omega}+1\right)\right] \\
& =\mathcal{M}_{322}^{(b)}+\mathcal{M}_{320}^{(b)}+\mathcal{M}_{32 R}^{(b)} \\
& \mathcal{M}_{322}^{(b)}=-\frac{8 i e^{4} \Gamma(4)}{\left(16 \pi^{2}\right)^{2}} \cdot \frac{1}{3} g^{\mu \nu} M_{c}^{2} \int_{0}^{1} \prod_{i=1}^{2} d x_{i} \int_{0}^{\infty} \prod_{i=1}^{2} \frac{d v_{i}}{\left(1+v_{i}\right)^{3}} \delta\left(1-\sum_{i=1}^{2} \frac{1}{1+v_{i}}\right) \int_{0}^{\infty} d u \\
& \frac{1}{\left[u+\frac{1}{\left(1+v_{1}\right)\left(1+v_{2}\right)}\right]^{3}} \frac{1}{\left(1+v_{1}\right)^{2}\left(1+v_{2}\right)^{2}} \\
& =-\frac{8 i e^{4} \Gamma(4)}{\left(16 \pi^{2}\right)^{2}} \frac{1}{36} g^{\mu \nu} M_{c}^{2} \\
& \mathcal{M}_{320}^{(b)}=-\frac{8 i e^{4} \Gamma(4)}{\left(16 \pi^{2}\right)^{2}} \frac{1}{3} g^{\mu \nu} p^{2} \int_{0}^{1} \prod_{i=1}^{2} d x_{i} \int_{0}^{\infty} \prod_{i=1}^{2} \frac{d v_{i}}{\left(1+v_{i}\right)^{3}} \delta\left(1-\sum_{i=1}^{2} \frac{1}{1+v_{i}}\right) \int_{0}^{\infty} d u \\
& \frac{1}{\left[u+\frac{1}{\left(1+v_{1}\right)\left(1+v_{2}\right)}\right]^{3}} \frac{1}{\left(1+v_{1}\right)^{2}\left(1+v_{2}\right)^{2}}\left(\ln \frac{M_{c}^{2}}{-p^{2}}-\gamma_{\omega}+1\right) \\
& \left\{\frac{1}{u+\frac{1}{\left(1+v_{1}\right)\left(1+v_{2}\right)}}\left[\frac{\left(x_{2}-x_{1}\right)^{2}}{\left(1+v_{1}\right)\left(1+v_{2}\right)}+\frac{x_{1}\left(1-x_{1}\right)}{1+v_{1}}+\frac{x_{2}\left(1-x_{2}\right)}{1+v_{2}}\right]\right. \\
& \left.-\frac{1}{\left[u+\frac{1}{\left(1+v_{1}\right)\left(1+v_{2}\right)}\right]^{2}} \frac{\left(x_{2}-x_{1}\right)^{2}}{\left(1+v_{1}\right)^{2}\left(1+v_{2}\right)^{2}}\right\} \\
& =-\frac{8 i e^{4} \Gamma(4)}{\left(16 \pi^{2}\right)^{2}} \frac{25}{36 \cdot 36} g^{\mu \nu} p^{2}\left(\ln \frac{M_{c}^{2}}{-p^{2}}-\gamma_{\omega}+1\right)
\end{aligned}
$$

Since we can prove that $\mathcal{M}_{32 R}^{(b)}$ is finite, the divergence structure we concern now is given by the addition of $\mathcal{M}_{322}^{(b)}$ and $\mathcal{M}_{320}^{(b)}$ :

$$
\mathcal{M}_{32}^{(b)} \sim-\frac{8 i e^{4} \Gamma(4)}{\left(16 \pi^{2}\right)^{2}} \frac{1}{36} g^{\mu \nu}\left[M_{c}^{2}+\frac{25}{36} p^{2}\left(\ln \frac{M_{c}^{2}}{-p^{2}}-\gamma_{\omega}+1\right)\right]
$$

Like $\mathcal{M}_{20}^{(b)}, \mathcal{M}_{30}^{(b)}$ contains three parts differentiating by their Lorentz structures: $\mathcal{M}_{301}^{(b)}$ represents part proportional to $p^{\mu} p^{\nu}, \mathcal{M}_{301}^{(b)}$ for $g^{\mu \nu} p^{2}$ and $\mathcal{M}_{303}^{(b)}$ for $g^{\mu \nu} \mu_{u}^{2}$. Let us first calculate 
$\mathcal{M}_{301}^{(b)}$

$$
\begin{aligned}
\mathcal{M}_{301}^{(b)=} & -\frac{8 i e^{4} \Gamma(4)}{\left(16 \pi^{2}\right)^{2}} \cdot \frac{1}{3} p^{\mu} p^{\nu} \int_{0}^{1} \prod_{i=1}^{2} d x_{i} \int_{0}^{\infty} \prod_{i=1}^{2} \frac{d v_{i}}{\left(1+v_{i}\right)^{3}} \delta\left(1-\sum_{i=1}^{2} \frac{1}{1+v_{i}}\right) \int_{0}^{\infty} d u \\
& {\left[\frac{1}{\left(1+v_{1}\right)\left(1+v_{2}\right)}-\left(\frac{x_{1}}{1+v_{1}}+\frac{x_{2}}{1+v_{2}}\right)\left(1-\frac{x_{1}}{1+v_{1}}-\frac{x_{2}}{1+v_{2}}\right)\right] } \\
& \frac{1}{\left[u+\frac{1}{\left(1+v_{1}\right)\left(1+v_{2}\right)}\right]^{3}}\left(\ln \frac{M_{c}^{2}}{\mu_{u}^{2}}-\gamma_{\omega}\right) \\
= & \mathcal{M}_{3010}^{(b)}+\mathcal{M}_{301 R}^{(b)} .
\end{aligned}
$$

Actual calculation of $\mathcal{M}_{3010}^{(b)}$ shows that the integral before $\left(\ln \frac{M_{c}^{2}}{p^{2}}-\gamma_{\omega}\right)$ is logarithmically divergent. So like the part $\mathcal{M}_{2030}^{(b)}, \mathcal{M}_{3010}^{(b)}$ also involves overlapping divergences.

$$
\begin{aligned}
\mathcal{M}_{3010}^{(b)}= & -\frac{8 i e^{4} \Gamma(4)}{\left(16 \pi^{2}\right)^{2}} \cdot \frac{1}{3} p^{\mu} p^{\nu} \int_{0}^{1} \prod_{i=1}^{2} d x_{i} \int_{0}^{\infty} \prod_{i=1}^{2} \frac{d v_{i}}{\left(1+v_{i}\right)^{3}} \delta\left(1-\sum_{i=1}^{2} \frac{1}{1+v_{i}}\right) \int_{0}^{\infty} d u \\
& \left.\frac{1}{\left(1+v_{1}\right)\left(1+v_{2}\right)}-\left(\frac{x_{1}}{1+v_{1}}+\frac{x_{2}}{1+v_{2}}\right)\left(1-\frac{x_{1}}{1+v_{1}}-\frac{x_{2}}{1+v_{2}}\right)\right] \\
= & -\frac{1}{\left.\left(u+\frac{1}{\left(1+e^{4} \Gamma(4)\right.}{ }^{2}\right)^{2}\right)^{2}} p^{\mu} p^{\nu}\left[\frac{5}{36}-\frac{1}{36}\left(\int_{0}^{\infty} \frac{d v_{1}}{1+v_{1}}+\int_{0}^{\infty}-\gamma_{\omega}\right)\right. \\
= & -\frac{8 i e^{4} \Gamma(4)}{\left(16 \pi^{2}\right)^{2}} p^{\mu} p^{\nu}\left[\frac{5}{36}\left(\ln \frac{M_{c}^{2}}{-p^{2}}-\gamma_{\omega}\right)-\frac{1}{18}\left(\ln \frac{M_{c}^{2}}{-p^{2}}-\gamma_{\omega}\right)\left(\ln \frac{M_{c}^{2}}{-p^{2}}-\gamma_{\omega}^{2}\right)\right.
\end{aligned}
$$

For the more challenging part $\mathcal{M}_{301 R}^{(b)}$, we follow the approach already used in the derivation of $\mathcal{M}_{203 R}^{(b)}$ in order to obtain only the asymptotic results. We first focus on the region $v_{1} \rightarrow \infty$ while $v_{2} \rightarrow 0$, the expression for $\mathcal{M}_{301 R}^{(b)}$ can be simplified to:

$$
\mathcal{M}_{301 R v_{1}}^{(b)} \sim-\frac{8 i e^{4} \Gamma(4)}{\left(16 \pi^{2}\right)^{2}} \cdot \frac{1}{3} p^{\mu} p^{\nu} \int_{0}^{1} \prod_{i=1}^{2} d x_{i} \int_{V}^{\infty} \frac{d v_{1}}{\left(1+v_{1}\right)^{3}} \int_{0}^{\infty} d u \frac{x_{2}\left(1-x_{2}\right)}{\left(u+\frac{1}{1+v_{1}}\right)^{3}} \ln \frac{x_{2}\left(1-x_{2}\right)}{u+\frac{1}{1+v_{1}}}
$$

where the integration over $v_{1}$ is confined in the region from $V \gg 1$ to infinity. It is easy to see that the simplified integral is essentially the same as that for $\mathcal{M}_{203 R}^{(b)}$, (C19), so we can straightforwardly write down the result for $\mathcal{M}_{301 R}^{(b)}$

$$
\mathcal{M}_{301 R}^{(b)} \sim \frac{8 i e^{4} \Gamma(4)}{\left(16 \pi^{2}\right)^{2}} \cdot \frac{1}{18} p^{\mu} p^{\nu}\left[\frac{13}{6}\left(\ln \frac{M_{c}^{2}}{-q_{o}^{2}(1+V)}-\gamma_{\omega}\right)-\frac{1}{2}\left(\ln \frac{M_{c}^{2}}{-q_{o}^{2}}-\gamma_{\omega}\right)^{2}+\frac{1}{2} \alpha_{\omega}\right],
$$

where we have already doubled the result for $\mathcal{M}_{301 R v_{1}}^{(b)}$ due to another asymptotic limit region $v_{2} \rightarrow \infty, v_{1} \rightarrow 0$, which gives the exactly same result. 
A very similar calculation as $\mathcal{M}_{301}^{(b)}$ can give us the results for $\mathcal{M}_{302}^{(b)}$,

$$
\begin{aligned}
& \mathcal{M}_{302}^{(b)}=-\frac{8 i e^{4} \Gamma(4)}{\left(16 \pi^{2}\right)^{2}} \cdot \frac{1}{3} g^{\mu \nu} p^{2} \int_{0}^{1} \prod_{i=1}^{2} d x_{i} \int_{0}^{\infty} \prod_{i=1}^{2} \frac{d v_{i}}{\left(1+v_{i}\right)^{3}} \delta\left(1-\sum_{i=1}^{2} \frac{1}{1+v_{i}}\right) \int_{0}^{\infty} d u \\
& \frac{1}{\left[u+\frac{1}{\left(1+v_{1}\right)\left(1+v_{2}\right)}\right]^{3}}\left\{-3 \frac{1}{\left[u+\frac{1}{\left(1+v_{1}\right)\left(1+v_{2}\right)}\right]^{2}} \frac{\left(x_{2}-x_{1}\right)^{2}}{\left(1+v_{1}\right)^{4}\left(1+v_{2}\right)^{4}}\right. \\
& +3 \frac{\left(x_{2}-x_{1}\right)}{\left(1+v_{1}\right)^{2}\left(1+v_{2}\right)^{2}}\left(\frac{x_{1}}{1+v_{1}}+\frac{x_{2}}{1+v_{2}}-\frac{1}{2}\right)\left(\frac{1}{1+v_{1}}-\frac{1}{1+v_{2}}\right) \frac{1}{u+\frac{1}{\left(1+v_{1}\right)\left(1+v_{2}\right)}} \\
& \left.+\frac{1}{2}\left(\frac{x_{1}}{1+v_{1}}+\frac{x_{2}}{1+v_{2}}\right)\left(1-\frac{x_{1}}{1+v_{1}}-\frac{x_{2}}{1+v_{2}}\right)\left[1-\frac{6}{\left(1+v_{1}\right)\left(1+v_{2}\right)}\right]\right\}\left(\ln \frac{M_{c}^{2}}{\mu_{u}^{2}}-\gamma_{\omega}\right) \\
& =\mathcal{M}_{3020}^{(b)}+\mathcal{M}_{302 R}^{(b)} \text {. } \\
& \mathcal{M}_{3020}^{(b)}=-\frac{8 i e^{4} \Gamma(4)}{\left(16 \pi^{2}\right)^{2}} \cdot \frac{1}{3} g^{\mu \nu} p^{2} \int_{0}^{1} \prod_{i=1}^{2} d x_{i} \int_{0}^{\infty} \prod_{i=1}^{2} \frac{d v_{i}}{\left(1+v_{i}\right)^{3}} \delta\left(1-\sum_{i=1}^{2} \frac{1}{1+v_{i}}\right) \int_{0}^{\infty} d u \\
& \frac{1}{\left[u+\frac{1}{\left(1+v_{1}\right)\left(1+v_{2}\right)}\right]^{3}}\left\{-3 \frac{1}{\left[u+\frac{1}{\left(1+v_{1}\right)\left(1+v_{2}\right)}\right]^{2}} \frac{\left(x_{2}-x_{1}\right)^{2}}{\left(1+v_{1}\right)^{4}\left(1+v_{2}\right)^{4}}\right. \\
& +3 \frac{\left(x_{2}-x_{1}\right)}{\left(1+v_{1}\right)^{2}\left(1+v_{2}\right)^{2}}\left(\frac{x_{1}}{1+v_{1}}+\frac{x_{2}}{1+v_{2}}-\frac{1}{2}\right)\left(\frac{1}{1+v_{1}}-\frac{1}{1+v_{2}}\right) \frac{1}{u+\frac{1}{\left(1+v_{1}\right)\left(1+v_{2}\right)}} \\
& \left.+\frac{1}{2}\left(\frac{x_{1}}{1+v_{1}}+\frac{x_{2}}{1+v_{2}}\right)\left(1-\frac{x_{1}}{1+v_{1}}-\frac{x_{2}}{1+v_{2}}\right)\left[1-\frac{6}{\left(1+v_{1}\right)\left(1+v_{2}\right)}\right]\right\}\left(\ln \frac{M_{c}^{2}}{-p^{2}}-\gamma_{\omega}\right) \\
& =-\frac{8 i e^{4} \Gamma(4)}{\left(16 \pi^{2}\right)^{2}} \cdot \frac{1}{3} g^{\mu \nu} p^{2}\left(\ln \frac{M_{c}^{2}}{-p^{2}}-\gamma_{\omega}\right)\left[-\frac{43}{16 \cdot 9}+\frac{1}{24}\left(\int_{0}^{\infty} \frac{d v_{1}}{1+v_{1}}+\int_{0}^{\infty} \frac{d v_{2}}{1+v_{2}}\right)\right] \\
& =-\frac{8 i e^{4} \Gamma(4)}{\left(16 \pi^{2}\right)^{2}} \cdot \frac{1}{3} g^{\mu \nu} p^{2}\left(\ln \frac{M_{c}^{2}}{-p^{2}}-\gamma_{\omega}\right)\left[-\frac{43}{16 \cdot 9}+\frac{1}{12}\left(\ln \frac{M_{c}^{2}}{-q_{o}^{2}}-\gamma_{\omega}\right)\right] \text {. }
\end{aligned}
$$

Note that the coefficient before $\left(\ln \frac{M_{c}^{2}}{-p^{2}}-\gamma_{\omega}\right)$ is divergent, which means that $\mathcal{M}_{302}^{(b)}$ also contains overlapping divergences. In order to deal with the resultant divergent integral $\mathcal{M}_{302 R}^{(b)}$, following the procedure used by $\mathcal{M}_{203 R}^{(b)}$ and $\mathcal{M}_{301 R}^{(b)}$, we need first to simplify the integral in the region $v_{1} \rightarrow \infty, v_{2} \rightarrow 0$

$$
\begin{aligned}
\mathcal{M}_{302 R v_{1}}^{(b)} & \sim \frac{8 i e^{4} \Gamma(4)}{\left(16 \pi^{2}\right)^{2}} \cdot \frac{1}{6} g^{\mu \nu} p^{2} \int_{0}^{1} \prod_{i=1}^{2} d x_{i} \int_{V}^{\infty} \frac{d v_{1}}{\left(1+v_{1}\right)^{3}} \int_{0}^{\infty} d u \frac{x_{2}\left(1-x_{2}\right)}{\left(u+\frac{1}{1+v_{1}}\right)^{3}} \ln \frac{x_{2}\left(1-x_{2}\right)}{u+\frac{1}{1+v_{1}}} \\
& \left.=-\frac{8 i e^{4} \Gamma(4)}{\left(16 \pi^{2}\right)^{2}} \cdot \frac{1}{72} g^{\mu \nu} p^{2}\left[\frac{13}{6}\left(\ln \frac{M_{c}^{2}}{-q_{o}^{2}(1+V)}-\gamma_{\omega}\right)-\frac{1}{2}\left(\ln \frac{M_{c}^{2}}{-q_{o}^{2}}-\gamma_{\omega}\right)^{2}+\frac{1}{2} \alpha_{\omega}\right] . \mathrm{C} 31\right)
\end{aligned}
$$

In order to obtain $\mathcal{M}_{302 R}^{(b)}$, the above result needs to be doubled to take into account another contribution from the region $v_{2} \rightarrow \infty, v_{1} \rightarrow 0$, which is given below:

$$
\mathcal{M}_{302 R}^{(b)} \sim-\frac{8 i e^{4} \Gamma(4)}{\left(16 \pi^{2}\right)^{2}} \cdot \frac{1}{36} g^{\mu \nu} p^{2}\left[\frac{13}{6}\left(\ln \frac{M_{c}^{2}}{-q_{o}^{2}(1+V)}-\gamma_{\omega}\right)-\frac{1}{2}\left(\ln \frac{M_{c}^{2}}{-q_{o}^{2}}-\gamma_{\omega}\right)^{2}+\frac{1}{2} \alpha_{\omega}\right] .
$$


For the accomplishment of the computation of $\mathcal{M}_{30}^{(b)}$, we have to calculate $\mathcal{M}_{303}^{(b)}$ :

$$
\begin{aligned}
\mathcal{M}_{303}^{(b)}= & -\frac{8 i e^{4} \Gamma(4)}{\left(16 \pi^{2}\right)^{2}} \cdot \frac{2}{3} g^{\mu \nu} p^{2} \int_{0}^{1} \prod_{i=1}^{2} d x_{i} \int_{0}^{\infty} \prod_{i=1}^{2} \frac{d v_{i}}{\left(1+v_{i}\right)^{3}} \delta\left(1-\sum_{i=1}^{2} \frac{1}{1+v_{i}}\right) \int_{0}^{\infty} d u \\
& \frac{1}{\left(1+v_{1}\right)^{2}\left(1+v_{2}\right)^{2}}\left(\ln \frac{M_{c}^{2}}{\mu_{u}^{2}}-\gamma_{\omega}\right) \\
& \left\{\frac{1}{u+\frac{1}{\left(1+v_{1}\right)\left(1+v_{2}\right)}}\left[\frac{\left(x_{2}-x_{1}\right)^{2}}{\left(1+v_{1}\right)\left(1+v_{2}\right)}+\frac{x_{1}\left(1-x_{1}\right)}{1+v_{1}}+\frac{x_{2}\left(1-x_{2}\right)}{1+v_{2}}\right]\right. \\
& \left.-\frac{1}{\left[u+\frac{1}{\left(1+v_{1}\right)\left(1+v_{2}\right)}\right]^{2}} \frac{\left(x_{2}-x_{1}\right)^{2}}{\left(1+v_{1}\right)^{2}\left(1+v_{2}\right)^{2}}\right\} \\
= & \mathcal{M}_{3030}^{(b)+\mathcal{M}_{303 R}^{(b)},}
\end{aligned}
$$

where $\mathcal{M}_{3030}^{(b)}$ represents the part proportional to the logarithmic divergence coming from $I_{0}^{R}$ while $\mathcal{M}_{303 R}^{(b)}$ the rest parts. It is a direct exercise to obtain $\mathcal{M}_{3030}^{(b)}$ and the result is:

$$
\mathcal{M}_{3030}^{(b)}=-\frac{8 i e^{4} \Gamma(4)}{\left(16 \pi^{2}\right)^{2}} \cdot \frac{25}{18 \cdot 36} g^{\mu \nu} p^{2}\left(\ln \frac{M_{c}^{2}}{-p^{2}}-\gamma_{\omega}\right)
$$

while part $\mathcal{M}_{303 R}^{(b)}$ can be proven finite. So the divergence structure of $\mathcal{M}_{303}^{(b)}$ is given by:

$$
\mathcal{M}_{303}^{(b)} \sim-\frac{8 i e^{4} \Gamma(4)}{\left(16 \pi^{2}\right)^{2}} \cdot \frac{25}{18 \cdot 36} g^{\mu \nu} p^{2}\left(\ln \frac{M_{c}^{2}}{-p^{2}}-\gamma_{\omega}\right)
$$

For the part $\mathcal{M}_{3 R}^{(b)}$, we can prove that most terms are finite for the integration of UVDP or Feynman parameters, except for the following one:

$$
\begin{aligned}
\mathcal{M}_{3 R 1}^{(b)}= & -\frac{8 i e^{4} \Gamma(4)}{16 \pi^{2}} \cdot \frac{1}{3}\left(g^{\mu \nu} p^{2}-2 p^{\mu} p^{\nu}\right) \int_{0}^{1} \prod_{i=1}^{2} d x_{i} \int_{0}^{\infty} \prod_{i=1}^{2} \frac{d v_{i}}{\left(1+v_{i}\right)^{3}} \delta\left(1-\sum_{i=1}^{2} \frac{1}{1+v_{i}}\right) \\
& \int_{0}^{\infty} d u\left[\frac{1}{\left(1+v_{1}\right)\left(1+v_{2}\right)}-\left(\frac{x_{1}}{1+v_{1}}+\frac{x_{2}}{1+v_{2}}\right)\left(1-\frac{x_{1}}{1+v_{1}}-\frac{x_{2}}{1+v_{2}}\right)\right] \\
& \frac{1}{\left[u+\frac{1}{\left(1+v_{1}\right)\left(1+v_{2}\right)}\right]^{3}} \frac{1}{16 \pi^{2}} \frac{1}{2 \mu_{u}^{2}} \mu_{u}^{2} \\
= & -\frac{8 i e^{4} \Gamma(4)}{\left(16 \pi^{2}\right)^{2}} \cdot \frac{1}{6}\left(g^{\mu \nu} p^{2}-2 p^{\mu} p^{\nu}\right) \int_{0}^{\infty} \prod_{i=1}^{2} \frac{d v_{i}}{\left(1+v_{i}\right)^{2}} \delta\left(1-\sum_{i=1}^{2} \frac{1}{1+v_{i}}\right) \\
& {\left[\frac{5}{12}-\frac{1}{12}\left(1+v_{1}\right)\left(1+v_{2}\right)\right] } \\
= & -\frac{8 i e^{4} \Gamma(4)}{\left(16 \pi^{2}\right)^{2}} \cdot \frac{1}{6}\left(g^{\mu \nu} p^{2}-2 p^{\mu} p^{\nu}\right)\left[\frac{5}{12}-\frac{1}{6}\left(\ln \frac{M_{c}^{2}}{-q_{o}^{2}}-\gamma_{\omega}\right)\right] \\
\sim & -\frac{8 i e^{4} \Gamma(4)}{\left(16 \pi^{2}\right)^{2}} \cdot\left(g^{\mu \nu} p^{2}-2 p^{\mu} p^{\nu}\right)\left(-\frac{1}{36}\right)\left(\ln \frac{M_{c}^{2}}{-q_{o}^{2}}-\gamma_{\omega}\right)
\end{aligned}
$$

By putting the divergence parts of the terms Eqs.(C1), (C5), (C9), (C12), (C15), (C17),

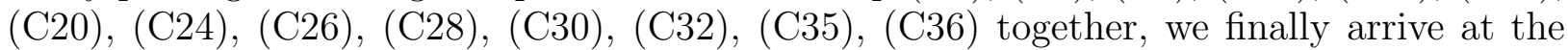


divergence behavior of the diagram $\mathcal{M}^{(b)}$.

$$
\begin{aligned}
\mathcal{M}^{(b)} \sim & -\frac{8 i e^{4}}{\left(16 \pi^{2}\right)^{2}}\left\{g^{\mu \nu} M_{c}^{2}\left(\ln \frac{M_{c}^{2}}{-q_{o}^{2}}-\gamma_{\omega}\right)+\frac{1}{6} g^{\mu \nu} p^{2}\left(\ln \frac{M_{c}^{2}}{-q_{o}^{2}}-\gamma_{\omega}\right)\right. \\
& +\left(g^{\mu \nu} p^{2}-p^{\mu} p^{\nu}\right) \cdot\left[\frac{1}{3}\left(\ln \frac{M_{c}^{2}}{-p^{2}}-\gamma_{\omega}\right)\left(\ln \frac{M_{c}^{2}}{-q_{o}^{2}}-\gamma_{\omega}\right)+\frac{19}{18}\left(\ln \frac{M_{c}^{2}}{-p^{2}}-\gamma_{\omega}\right)\right] \\
& \left.-\frac{1}{3}\left(\ln \frac{M_{c}^{2}}{-q_{o}^{2}}-\gamma_{\omega}\right)+\frac{1}{3}\left[\frac{13}{6}\left(\ln \frac{M_{c}^{2}}{-q_{o}^{2}(1+V)}-\gamma_{\omega}\right)-\frac{1}{2}\left(\ln \frac{M_{c}^{2}}{-q_{o}^{2}}-\gamma_{\omega}\right)^{2}+\frac{1}{2} \alpha_{\omega}\right]\right\}
\end{aligned}
$$

\section{Appendix D: Derivation of the Integration $\int_{0}^{\infty} \frac{d v_{2}}{1+v_{2}} \ln \left(1+v_{2}\right)$}

Recall that in the above derivation of the two-loop massless QED photon vacuum polarization diagrams we encountered a new parameter integral

$$
\int_{0}^{\infty} \frac{d v_{2}}{1+v_{2}} \ln \left(1+v_{2}\right)
$$

which does not appear at one-loop level. Thus, for completion of our calculation, in this section we shall derive its regulated result with the LORE method.

With the same philosophy as before, we would like to transform this UVDP parameter integral to a momentum-like one. In order to do so, we just need to multiply a free energy scale $-q_{o}^{2}$ in the numerator and denominator simultaneously,

$$
\begin{aligned}
\int_{0}^{\infty} \frac{d v_{2}}{1+v_{2}} \ln \left(1+v_{2}\right) & =\int_{0}^{\infty} \frac{d q^{2}}{q^{2}-q_{o}^{2}} \ln \left(q^{2}-q_{o}^{2}\right)-\int_{0}^{\infty} \frac{d q^{2}}{q^{2}-q_{o}^{2}} \ln \left(-q_{o}^{2}\right) \\
& =I_{1}-I_{2},
\end{aligned}
$$

where we have defined $q^{2}=-q_{o}^{2} v_{2}$ and separated the integral into two parts $I_{1}$ and $I_{2}$. The integration $I_{2}$ can be easily worked out with the LORE method since it is exactly the one encountered in the one-loop calculations. The result of $I_{2}$ is

$$
I_{2}=\ln \left(-q_{o}^{2}\right)\left[\ln \frac{M_{c}^{2}}{\mu_{q}^{2}}-\gamma_{\omega}+y_{0}\left(\frac{\mu_{q}^{2}}{M_{c}^{2}}\right)\right]
$$

where we define $\mu_{q}^{2} \equiv \mu_{s}^{2}-q_{o}^{2}$.

The integration $I_{1}$ is really new and requires us to calculate more carefully. In the following, we shall give the detailed derivation of this integration in the LORE method. As usually done in the LORE method, we need to apply a series of regulators to the integral:

$$
I_{1}=\lim _{N \rightarrow \infty} \sum_{l=0}^{N} c_{l}^{N} \frac{d q^{2}}{q^{2}+\hat{\mathcal{M}}_{l}^{2}} \ln \left(q^{2}+\hat{\mathcal{M}}_{l}^{2}\right),
$$

where we defined $\hat{\mathcal{M}}_{l}^{2}=-q_{o}^{2}+\mu_{s}^{2}+l M_{R}^{2}=\mu_{q}^{2}+l M_{R}^{2}$. With the help of the following equality:

$$
\lim _{N \rightarrow \infty} \sum_{l=0}^{N} c_{l}^{N} l^{n}=0 \quad \text { with } \quad n=0,1, \ldots, N-1
$$


we can easily work out the above integration:

$$
\begin{aligned}
I_{1} & =\lim _{N \rightarrow \infty} \sum_{l=0}^{N} c_{l}^{N}\left(-\frac{1}{2}\right) \ln ^{2} \hat{\mathcal{M}}_{l}^{2}=\left(-\frac{1}{2}\right) \lim _{N \rightarrow \infty} \sum_{l=0}^{N} c_{l}^{N} \ln ^{2}\left(\mu_{q}^{2}+l M_{R}^{2}\right) \\
& =\left(-\frac{1}{2}\right)\left\{\ln ^{2} \mu_{q}^{2}+\lim _{N \rightarrow \infty} \sum_{l=1}^{N} c_{l}^{N} \ln ^{2}\left(\mu_{q}^{2}+l M_{R}^{2}\right)\right\} \\
& =\left(-\frac{1}{2}\right)\left\{\ln ^{2} \mu_{q}^{2}+\lim _{N \rightarrow \infty} \sum_{l=1}^{N} c_{l}^{N}\left[\ln \left(1+\frac{\mu_{q}^{2}}{l M_{R}^{2}}\right)+\ln l+\ln M_{R}^{2}\right]^{2}\right\} \\
& =\left(-\frac{1}{2}\right)\left\{\ln ^{2} \mu_{q}^{2}+\lim _{N \rightarrow \infty} \sum_{l=1}^{N} c_{l}^{N}\left[\ln M_{c}(N)^{2}-\gamma_{\omega}(N)+\ln l+\sum_{l^{\prime}=1}^{N} c_{l^{\prime}}^{N} \ln l^{\prime}+\ln \left(1+\frac{\mu_{q}^{2}}{l M_{R}^{2}}\right)\right]^{2}\right\} \\
& =\frac{1}{2}\left\{\left(\ln M_{c}^{2}-\gamma_{\omega}\right)^{2}-\ln ^{2} \mu_{q}^{2}-\alpha_{\omega}+2 \ln \mu_{q}^{2} \cdot y_{0}\left(\frac{\mu_{q}^{2}}{M_{c}^{2}}\right)+y_{0}^{\prime}\left(\frac{\mu_{q}^{2}}{M_{c}^{2}}\right)\right\}
\end{aligned}
$$

where we have introduced two series:

$$
M_{c}^{2}(N) \equiv \sum_{l=1}^{N} c_{l}^{N}(l \ln l) M_{R}^{2}, \quad \text { and } \quad \gamma_{\omega}(N) \equiv \sum_{l=1}^{N} c_{l}^{N} \ln l+\ln \left[\sum_{l^{\prime}}^{N} c_{l^{\prime}}^{N}\left(l^{\prime} \ln l^{\prime}\right)\right]
$$

both of which in the limit of $N \rightarrow \infty$ can be shown [3] to have finite limits

$$
M_{c}^{2}=\lim _{N \rightarrow \infty} M_{c}^{2}(N), \quad \text { and } \quad \gamma_{\omega}=\lim _{N \rightarrow \infty} \gamma_{\omega}(N) .
$$

We also define a new function

$y_{0}^{\prime}\left(\frac{\mu_{q}^{2}}{M_{c}^{2}}\right)=-\lim _{N, M_{R}^{2}} \sum_{l=1}^{N} c_{l}^{N} \ln \left(1+\frac{\mu_{q}^{2}}{l M_{R}^{2}}\right)\left[2\left(\ln l+\ln \frac{M_{c}^{2}(N)}{\mu_{q}^{2}}-\gamma_{\omega}(N)+\sum_{l^{\prime}=1}^{N} c_{l^{\prime}}^{N} \ln l^{\prime}\right)+\ln \left(1+\frac{\mu_{q}^{2}}{l M_{R}^{2}}\right)\right]$,

which can be seen that when the UV scale $M_{c}^{2}$ tends to infinity the function $y_{0}^{\prime}\left(\frac{\mu_{q}^{2}}{M_{c}^{2}}\right)$ vanishes since the expansion of $\ln \left(1+\frac{\mu_{q}^{2}}{l M_{R}^{2}}\right)$ is of the order of $O\left(\frac{\mu_{q}^{2}}{M_{R}^{2}}\right)$. The constant $\alpha_{\omega}$ is defined as

$$
\alpha_{\omega} \equiv \lim _{N \rightarrow \infty}\left[\sum_{l=1}^{N} c_{l}^{N}(\ln l)^{2}+\left(\sum_{l=1}^{N} c_{l}^{N} \ln l\right)^{2}\right] .
$$

It can be shown from our numerical calculation that $\alpha_{\omega}$ is finite and is $\alpha_{\omega}=1.62931 \ldots$.

By combining the expression $I_{1}$ and $I_{2}$, we can give our final regularized result

$$
\begin{aligned}
& \int_{0}^{\infty} \frac{d v_{2}}{1+v_{2}} \ln \left(1+v_{2}\right)=I_{1}-I_{2} \\
= & \frac{1}{2}\left[\left(\ln \frac{M_{c}^{2}}{\mu_{q}^{2}}-\gamma_{\omega}\right)^{2}+2 \ln \frac{\mu_{q}^{2}}{-q_{o}^{2}}\left(\ln \frac{M_{c}^{2}}{\mu_{q}^{2}}-\gamma_{\omega}\right)-\alpha_{\omega}+2 \ln \frac{\mu_{q}^{2}}{-q_{o}^{2}} y_{0}\left(\frac{\mu_{q}^{2}}{M_{c}^{2}}\right)+y_{0}^{\prime}\left(\frac{\mu_{q}^{2}}{M_{c}^{2}}\right)\right] .(\mathrm{D})
\end{aligned}
$$

When we set the IR scale $\mu_{s}^{2}$ to 0 which can be viewed as the IR cutoff in the LORE method, then the regulated integral can be simplifies to:

$$
\int_{0}^{\infty} \frac{d v_{2}}{1+v_{2}} \ln \left(1+v_{2}\right)=\frac{1}{2}\left[\left(\ln \frac{M_{c}^{2}}{-q_{o}^{2}}-\gamma_{\omega}\right)^{2}-\alpha_{\omega}+y_{0}^{\prime}\left(\frac{-q_{o}^{2}}{M_{c}^{2}}\right)\right] .
$$


If we further take the limit $M_{c} \rightarrow \infty$, the last term $y_{0}^{\prime}\left(\frac{-q_{o}^{2}}{M_{c}^{2}}\right) \rightarrow 0$ also, which gives the result we use in our previous calculations.

[1] D. Huang and Y. -L. Wu, Eur. Phys. J. C 72, 2066 (2012) arXiv:1108.3603 [hep-ph]].

[2] G. 't Hooft and M. J. G. Veltman, Nucl. Phys. B 44, 189 (1972).

[3] Y. L. Wu, Int. J. Mod. Phys. A 18, 5363 (2003) arXiv:hep-th/0209021.

[4] Y. L. Wu, Mod. Phys. Lett. A 19, 2191 (2004) arXiv:hep-th/0311082.

[5] J. Bjorken and S. Drell, USA: McGraw-Hill Book Company (1965) $396 p$

[6] M. E. Peskin and D. V. Schroeder, Reading, USA: Addison-Wesley (1995) 842 p

[7] C. Itzykson and J. B. Zuber, New York, USA: Mcgraw-hill (1980) 705 P.(International Series In Pure and Applied Physics)

[8] R. Jost and J. M. Luttinger, Helv. Phys. Acta, 23, 201 (1950) 